\title{
4 Anachronismus und Verwandtes im Urteil der Ovid-Philologie
}

\section{1 „Dem Dichter ist [...] die Phantasie durchgegangen“ - ein Forschungsüberblick}

Das im Titel stehende Zitat ${ }^{1}$ aus Bömers „,Real-Encyklopädie“ der Metamorphosen“2 ist ein prägnantes Beispiel für die Perspektive, mit der Ovids Verwandlungssagen in ihrer ambigen und unkonventionellen Darbietung immer wieder bewertet wurden und z.T. noch werden. Von der an Bömer schätzenswerten Deutlichkeit im Urteilen abgesehen, ${ }^{3}$ ist es doch erstaunlich, dass sich die Phantasie gerade eines solchen Dichters als übertrieben erweisen sollte, die von diesem selbst bzw. dessen Sprecher-Ich als fecunda licentia vatum stolz als ins Unermessliche gehend proklamiert wird. ${ }^{4}$ Ebenso bemerkenswert ist, dass der an dieser Stelle entdeckte Mangel hauptsächlich daran liegt, dass „ein Anachronismus“ vorliegt und „der Kritik [...] außerdem ein Blick auf die sozialen Verhältnisse nicht stand[hält]“5; ganz so, als ob es Ovids Anspruch an dieser Stelle wäre, ein wahrheitsgemäßes Abbild der sozialen Verhältnisse zur wohlweißlich mythischen Gründungszeit Milets zu geben. ${ }^{6}$ Dass dem Dichter „die Phantasie durchgegangen“ sei, legt somit auch Zeugnis davon ab, wie sehr die ästhetische Wirkung daran bemessen wird, dass einer ganz spezifischen Erwartung, sei diese nun interpretatorisch geboten oder nicht, entsprochen wird. ${ }^{7}$ Bei deren Verfehlung ist folgerichtig auch die Darstellung kritikwürdig und ein Zeichen von Nachlässigkeit oder Übertreibung. ${ }^{8}$

Aus diesem Grund auch ist die Beurteilung der Anachronismen ein Spiegel der Deutung der „Metamorphosen“ überhaupt, da sich am Extremfall nur die grundsätzlichen Schwächen und Stärken einer Methodik und ihrer interpretatorischen Prämissen offenbaren. Der nun folgende, weitgehend chronologische Überblick wird dies bestätigen. ${ }^{9}$

1 Bömer 1977, ad met. IX, 718, zu der an dieser Stelle sehr deutlichen Aktualisierung (dort als Anachronismus bezeichnet).

2 Klein 2005, 190.

3 Vgl. Dräger 2006, 1178.

4 S. o. Kap. 3.1.2.

5 Bömer 1977, ad met. IX, 718.

6 Thematisch geht es hier um den Hermaphroditismus bei Iphis (Ov. met. IX, 666-797).

7 Zur Feststellung, dass dies mehr Rezeption als Interpretation ist, s. o. S. 70, Anm. 238.

8 Diese wird von Bömer, ähnlich wie Cicero in seinem Kommentar zur Begegnung von Numa und Pythagoras (s. o. Kap. 2.2.1.1), via Genealogien nachgerechnet, als historisches Faktum gesetzt und offenbar als die zu erfüllende Norm für das künstlerische Abbild festgelegt, obwohl eine Historizität in der erzählten Welt aus gutem Grund keine Rolle spielt.

9 Auch wenn ein überwiegender Teil dieser Besprechung als ein „wearying chronicle of outmoded, predominantly German findings“ (Holzberg 2005, 697) aufgefasst werden könnte, erfordert es allein der 


\subsubsection{Arbeiten bis in die 1930er Jahre}

Die einzige Monographie, die ausschließlich den Anachronismen gewidmet ist, stellt bislang Eberts 1888 erschienenes Buch „Der Anachronismus in Ovids Metamorphosen“ dar. Dadurch könnte der Eindruck entstehen, dass dieses Thema trotz stetigen Anwachsens der Forschungsliteratur ${ }^{10}$ so zufriedenstellend behandelt worden ist, dass es nur mehr durch Einzelbeobachtungen ergänzt oder bei Überschneidungen mit anderen Phänomenen diskutiert werden müsste. ${ }^{11}$ Bei genauerem Hinsehen zeigen sich jedoch gravierende Probleme.

Ausgehend von der knappen Definition des Anachronismus als „Verstoss gegen die Zeitverhältnisse“12 beginnt Ebert einen gelehrten Streifzug durch die Literaturgeschichte und setzt ein bei der Geschichtsschreibung (Livius und Prokop), um ganz ähnlich wie Cicero in „De legibus“ auf die verschiedenartige Verpflichtung zur Wahrheit bei einem Historiographen und einem Dichter hinzuweisen. ${ }^{13}$ Wichtig sei jedoch, so sein Fazit, auch für den Dichter, „Mass zu halten“ und das „Abweichen von der historischen Wahrheit“ nicht so weit zu führen, dass „der Genuss des Kunstwerkes beeinträchtigt wird. “14

So wird zwar die künstlerische Freiheit stets betont und sogar auf die besondere Ausprägung des „geschichtlichen Sinns“ in der eigenen Zeit hingewiesen, weswegen eine vermeintlich anachronistische Darstellung in früheren Zeiten aus ihrem Kontext heraus zu verstehen und nicht generell fehlerhaft ist; ${ }^{15}$ jegliches Beispiel wird aber dennoch aus der gegenwärtigen, historistischen Position als falsch oder vertretbar ,in allen Abstufungen von den unmerklichsten bis zu den schreiendsten“"16 bewertet. Allein, dass historische Wahrheit eine für den Kunstgenuss ausschlaggebende Kategorie ist, lässt erahnen, wie sehr dieser Maßstab als ein der Ästhetik des Textes überlegener vorausgesetzt ist, ${ }^{17}$ von dem ausgehend die doch sehr überzeugt wirkenden Urteile abgegeben werden. ${ }^{18}$

Umstand, dass diese vermeintlich aus der Mode gekommenen Argumente oder Methoden nach wie vor bzw. immer noch (und über Länder- und Sprachgrenzen hinweg) Anhänger finden oder unhinterfragt wiederholt werden, ein solches „chronicle“ zur Niederschrift zu bringen.

10 S. dazu o. S. 1, Anm. 2.

11 Noch Wheeler stellt beispielsweise in einer Anmerkung fest: „The most comprehensive study of Ovid's Romanizing point of view is Ebert“ (Wheeler 1999a, 237 (Anm. 7)). Zur Romanization s. o. Kap. 3.3.3.1 und u. Kap. 7.2.2.1c.

12 Ebert 1888, 1. Andere Umschreibungen hierfür sind u.a. „mit den Zeitverhältnissen nicht übereinstimmen“ (S. 1), „Übertragung von Neuerem auf frühere Zeiten“ (S. 1), „zeitliche Verschiebungen“ (S. 2). Der allgemeine Überblick geht bis Seite 12.

13 Vgl. ebd., 1f. Von der Konzeption ähnlich und ebenso mit antiken Beispielen Braun 1942.

14 Ebert 1888, $2-4$.

15 Vgl. ebd.

16 Ebd., 4.

17 Dies ist implizit immer vorausgesetzt, wird aber z. T. auch direkt geäußert, z. B. auf Seite 3 oder auf Seite 12, wo es in Anlehnung an Gustav Freytag „als Fortschritt unserer Zeit“ gilt, dass „,man es mit der scenischen Ausstattung genauer nimmt“. Vgl. auch Tronchet 1998, 361. 
Dass Ebert die bislang umfassendste Zusammenstellung an Anachronismen vorgelegt hat, ist sicherlich seiner „eigentlichen Aufgabe“ zu verdanken, „den Anachronismus in Ovids Metamorphosen nachzuweisen. " ${ }^{\text {"19 }}$ Diese beinahe detektivische Haltung gründet sich zum einen auf der Vorstellung von einer organischen Einheit des griechischen Mythos und zum anderen in der Ansicht, diese Einheit strikt historisch bewerten und Fremdes, in diese Welt Eindringendes identifizieren zu können. ${ }^{20}$ Der Mythos, das Resultat einer jahrhundertelangen und sogar sehr gut in der griechischen Dichtung und Prosa nachvollziehbaren Aneignung, Kritik und Umdeutung, wird gegenüber Ovids Adaption als etwas Abgeschlossenes betrachtet, das allein dadurch, dass es vermeintlich griechisch ist, älter ist. ${ }^{21}$ So unspezifisch allein dies ist, so erweist sich dies als umso trügerischerer Ausgangspunkt für interpretatorisches Arbeiten, wenn schon die Dichtung selbst sich diesem Anspruch, und damit auch den Erwartungen eines dem Historismus entstammenden Rezipienten, wiederholt entzieht. ${ }^{22}$

Allgemeine Wertungen, wonach z. B. ,in gewaltsamer, wenig poetischer Weise“ ${ }^{\text {223 }}$ erzählt werde, sind neben einigen eher willkürlichen methodischen Festlegungen die Folge: Anachronismen nach der oben erarbeiteten Definition sind etwa als „unerlaubte Widersprüche“ von Vornherein ausgeschlossen und deren Zusammenstellung „wäre wertlos“, obwohl ihre Häufigkeit herausgestellt bzw. die eigene Fähigkeit, diese zahlreich nachweisen zu können, wie selbstverständlich impliziert wird. ${ }^{24}$ Dass im Gegenteil aber die Häufigkeit eines Befundes ein Indiz für dessen Zugehörigkeit zum

18 Um nur eine Auswahl zu geben: „Störend muss z. B. der Anachronismus wirken“ (S. 3), „So dürfte denn auch dem Dichter der Tadel nicht erspart bleiben“ (S. 3), „Was sich Dichter hier im kleinen Masstabe erlauben, erlaubt sich die Sage im grossen“ (S. 5). Nicht verwunderlich ist es, dass mehr oder minder zeitgleich ähnliche Abhandlungen zum Anachronismus bei anderen lateinischen Dichtern und Schriftstellern erschienen sind, s. dazu Stemplinger 1956, 103 (Anm. 3).

19 Ebert 1888, 12.

20 Dies geht dann sogar so weit, dass der Umstand, dass in den „Metamorphosen“ die meisten der dargestellten griechischen und römischen Sitten nicht unterscheidbar sind, mit der gemeinsamen Stammesverwandtschaft (!) der Griechen und Römer begründet wird, die auf die Zeit „vor ihrer Abzweigung von dem gemeinsamen Urvolke“ zurückgehe und noch durch den späteren Kulturtransfer von Griechenland nach Rom bestärkt worden sei. Vgl. ebd., $13 \mathrm{f}$.

21 Als ob es in der augusteischen Zeit keine griechische Kultur im Mittelmeerraum mehr gegeben hätte. Dazu wohl am passendsten Otis ${ }^{2} 1970$, 124: „Ovid is not simply contrasting Greek and Roman ideas. He has cast all his material - whether Roman-Virgilian or Hellenic-Hellenistic in its sources into much the same mould and style. [...] To put it another way: Ovid's Jupiter is not Roman when he presides over the gods and Greek when he seduces Europa.“ Vgl. auch Holzberg 1997, 157.

22 Wie groß diese Kluft sein kann, führt folgende Aussage emblematisch vor: „In der heroischen Zeit war es allgemeine Sitte der Griechen“ (Ebert 1888, 25). Ähnlich auch bei Haupt/Ehwald (s. o. S. 71f., Anm. 241) und Bömer ${ }^{2} 2006$, ad met. XII, 155f.: „In heroischer Zeit speiste man sitzend.“

23 Ebert 1888, 15.

24 Ebd., 14. Er bezieht sich dort auf die von ihm vorher geäußerte Festlegung: „Vor allem ist nie, auch in der Kunst nicht, gestattet, dass das Werk in sich einen Widerspruch enthält“ (S. 3). Warum dann ein Zeitverstoß im einen Fall gestattet und im anderen Fall nicht (er spricht auch dort von „Verstösse gegen die Zeit“ S. 14), erschließt sich daraus nicht. Im Register bei Siebelis/Polle ${ }^{8} 1875$ ist ebenso - bei nur einem einzigen Anachronismus-Beispiel - von einem „Widerspruch in der Sage“ die Rede. 
Textganzen wäre, macht die Klassifizierung als „unerlaubt“ schon aus sich heraus zweifelhaft. $^{25}$

Methodisch ungenau wirkt gleichfalls der Umgang mit dem, was als poetisch erlaubte Anachronismen angesehen wird: Epische Gleichnisse seien zwar „nicht so beweiskräftig“ wie andere Beispiele, werden aber angeführt, weil „etwas in eine Zeit getragen wird, was ihr fremd ist“ ${ }^{\star 26}$, wobei an der jeweiligen Stelle dann darauf hingewiesen sei, dass es sich um einen Vergleich handle. ${ }^{27}$ Auf gleiche Weise werden temporal markierte Aussagen, die der Erzählerstimme klar zugewiesen sind, ${ }^{28}$ für diese Art der Beweisführung in Beschlag genommen.

Letztlich bekräftigen die sehr deutlichen, aber nicht ausreichend begründeten Wertungen vornehmlich den eigenen Standpunkt des literaturhistorisch bzw. historisch Urteilenden. ${ }^{29}$ Eine tatsächliche Beschreibung, geschweige denn Deutung der Einzelbeispiele und eine Untersuchung der Frage, wie das Phänomen Anachronismus/Aktualisierung in seiner Stellung für die „Metamorphosen“ im Ganzen zu bewerten ist, bleibt aus. ${ }^{30}$

Blickt man in weitere aus dieser Zeit stammende Abhandlungen, stellt sich ein sehr ähnlicher Eindruck hinsichtlich Methodik und Betrachtungsweise ein. In der von Moritz Haupt begonnenen und im Laufe der Jahrzehnte mehrmals von weiteren Herausgebern wiederaufgelegten „Metamorphosen“-Ausgabe ist u. a. von „starkem“ oder „kühnem Anachronismus“31 die Rede und wie auch bei Ebert ist dieser Standpunkt des Urteilens ein strikt historischer, der jede Vermischung, Übertragung oder schlicht

25 Gerade wenn nicht geklärt ist, ab wann und von wem diese Erlaubnis dann zu erteilen wäre.

26 Ebert 1888, 19.

27 Dies unterbleibt aber teilweise bzw. wird mit Ausdrücken wie „anspielen“ (u.a. S. 18) oder „sehr offen gegenüber stellen“ (S. 15) umschrieben.

28 Auf diesen Unterschied weist schon Magnus in einer Rezension der „Metamorphosen“-Ausgabe von Haupt/Ehwald hin, vgl. Magnus 1916, 1616. Womöglich stand Ebert hinsichtlich der Gleichnisse der Sichtweise Moritz Haupts (s.u.) nahe, in einer Anmerkung weist er z. B. darauf hin, dass es u.a. in dessen Ausgabe vielfache Hinweise auf Anachronismen gebe. Vgl. Ebert 1888, 15. U. a. auch bei Frécaut 1972, 64 f., findet sich diese Auffassung über vermeintlich anachronistische Gleichnisse.

29 Vgl. auch Solodow 1988, 6.

$30 \mathrm{Ob}$ der Vergleich bzw. die Feststellung der Ähnlichkeit von Ovid mit den Dichtern mittelalterlicher Heldenepik, auf die Eberts „Nachweis“ hinausläuft - dies wird am Beginn der Besprechung der „Metamorphosen“ behauptet und als interpretatorischer Abschluss wiederholt (vgl. Ebert 1888, 15 und 35) -, überhaupt zweckdienlich ist, ohne vorher die Ästhetik der jeweiligen Texte in Augenschein genommen zu haben, muss entschieden bezweifelt werden.

31 Haupt/Korn ${ }^{5} 1966,120$ (ad IX, 719) und 380 (ad XIV, 324 f.). In der Ausgabe von Siebelis/Polle (Band I: ${ }^{10} 1878$; Band II: $\left.{ }^{8} 1875\right)$ wird an den Stellen, die im Register als „Anachronismen“ angeführt werden, deutlich zurückhaltender, hauptsächlich deskriptiv u. a. von Entlehnung aus einer späteren Zeit (Band I, 111 (ad met. VI, 221f.)) oder von einer „Einmischung römischen Gebrauchs“ gesprochen (Band II, 76 (ad met. XIII, 252)). Interessant ist aber die an einer Stelle für den sog. Anachronismus bemühte Erklärung als „Ausnahmefall“, der durch die „Nothlage“ im Geschehenszusammenhang bedingt sei (Band I, S. 81 (ad met. IV, 705)). Auch wenn sich diese Begründung hier sehr gut anzubieten scheint, ist sie doch für das Gesamtgedicht kaum aufrechtzuerhalten, weil Ovid über die ganzen „Metamorphosen“ hinweg diese Aktualisierung (bei der Mitgift) vornimmt. Dies bespricht auch Ebert 1888, 22. 
Aktualisierung der in all ihren Facetten als historisch empfundenen heroischen Zeit als Abweichen von der Wahrheit ansieht - ungeachtet verschiedener Erzählebenen oder Zeitmarkierungen. ${ }^{32}$ Eine eher nebenbei geäußerte Bemerkung in der Einleitung bringt dies gut auf den Punkt: „Er reiht, durch kleine Widersprüche und Anachronismen mit Recht ungestört, seine Erzählungen zu einem chronologischen Faden auf;““33 Die Widersprüche, bei denen Ebert andeutet, sie könnten sich „in grösserer Zahl“34 nachweisen lassen, müssen klein und wie die Anachronismen unberechtigt bleiben, um das vermeintlich wichtigere Prinzip der Chronologie nicht zu sehr als widersprüchlich ausweisen oder es in seiner absoluten Gültigkeit einschränken zu müssen. ${ }^{35}$

Ebenso als unbedeutend und in geringer Anzahl vorhanden bezeichnet Peters in einem knappen Absatz zu dieser Thematik diejenigen Fälle, in denen bei den „facta“ eine Vermischung von Römischem mit Griechischem erkennbar sei. ${ }^{36}$ Gleichnisse zählen genauso dazu wie andere Beispiele. Viel schwerwiegender sei es für ihn jedoch, dass die Färbung bzw. die Ausgestaltung der Gefühle und der Geschichten römisch sei und dass die Götter so wie der Dichter dächten und fühlten, aber keine göttliche Wesensart zur Schau stellten, sondern sich eher wie römische Adlige verhielten. ${ }^{37}$

32 Hierzu zählt der bereits von Magnus kritisierte Kommentar (s. o. S. 93, Anm. 28) zu einem Gleichnis, wobei auch auf weitere einschlägige Gleichnisse verwiesen wird. Vgl. Haupt ${ }^{11} 1969,153$ (ad met. III, 111). Eine Richtigstellung mit dem Hinweis auf Magnus hat von Albrecht unter seiner Herausgeberschaft bei der Stelle angefügt. Vgl. hierzu auch von Albrecht 1981, 2332.

33 Haupt ${ }^{11} 1969$, 9. S. hierzu auch die Kritik an einer anderen ähnlichen Vorstellung (Pfeiffer 1934, 48: „aber auch die ,Metamorphosen“ [...] hielten vom ersten Wandel des Chaos in den Kosmos bis zum letzten Wandel des politischen Chaos in den neuen Kosmos der augusteischen Ära im großen die zeitliche Abfolge fest") von Schmidt 1991, $41 \mathrm{f}$.

34 Ebert 1888, 14.

35 Und das, obwohl das Aufspüren dieser Widersprüche implizit den eigenen Scharfsinn unter Beweis zu stellen scheint, was an einigen Stellen nicht versäumt wird unter Beweis zu stellen. Vgl. Haupt ${ }^{11} 1969$ : „Dabei hat er nicht bedacht, oder auf Leser gerechnet, die über der Freude an seinen mannigfachen Erzählungen den Widerspruch übersehen“ (95, ad met. II, 171f.), „Ovid vergißt“ (110, ad met. II, 377), „erinnert er sich nicht daran“ (112, ad met. II, 401-530).

36 Vgl. Peters 1908, 79: „Uno verbo tangam, quomodo Ovidius Romanas res cum Graecis miscuerit. [...] Sed haec tantum pauca nec magni momenti esse vides ita ut hac in re etiamsi poeta confusionem temporum non vitaverit, tamen re vera eam haud saepe admiserit.“ (,Kurz möchte ich darauf zu sprechen kommen, wie Ovid römische Dinge mit griechischen vermischt. [...] Aber dass diese Dinge nur wenige und von geringem Gewicht sind, sieht man daran, dass er dabei, auch wenn der Dichter eine Vermengung der Zeiten nicht gemieden hat, diese dennoch nicht häufig beging.').

37 Vgl. ebd., 79f.: „Multo gravius est quod totus color affectuum et historiarum Romanus est, quod dei et deae Ovidianae idem sentiunt et putant atque poeta, quod minime naturam deorum prae se ferunt, sed potius mores iuvenum divitum et nobilium Romanorum.“ (,Viel schwerwiegender ist es, dass die ganze Färbung der Affekte und der Einzelgeschichten römisch ist, dass die ovidischen Götter und Göttinnen dasselbe fühlen und meinen wie der Dichter, dass sie sehr wenig ein göttliches Wesen an den Tag legen, sondern viel eher die Gewohnheiten der reichen und vornehmen römischen Jünglinge.`). Ebenso werden bei Rohde 1929, 13, Aktualisierungen im selben Zug wie die Bildebene von 
Anachronismen verdeutlichen in dieser Beurteilung offenbar den fehlenden Respekt Ovids sowohl gegenüber der vermeintlichen historischen Wahrheit als auch der paganen Götterwelt, wobei in der Zusammenschau mit Haupt und Ebert gut ersichtlich wird, wie subjektiv die jeweilige zahlenmäßige und ästhetische Gewichtung letztlich ist.

Das Phänomen selbst wird dabei immer nur so vage definiert oder so weit ausdifferenziert, wie es die eigene Themenstellung erfordert, und allein durch sein teils als schwerwiegend, teils als geringfügig bewertetes Abweichen von einer idealtypisch vorausgesetzten Welt, nicht aber als an sich bedeutsam besprochen. Am deutlichsten zeigt sich dies bei Kroll, der dem Kapitel „Originalität und Nachahmung“ einen Exkurs zum Anachronismus anhängt, hierzu aber ausschließlich Vergils „Aeneis“ bespricht und nur im letzten Absatz einige weitere Beispiele nennt. Folgendes wird aber gewissermaßen warnend vorausgeschickt: „Beispiele aus Späteren zu häufen hat wenig Zweck: Sie mischen unbedenklich Griechisches und Römisches und vereinigen homerische und vergilische Motive. “38 Zwar ist der Fokus durch die Diskussion der Originalität römischer Dichtung von vornherein auf den Unterschied zum griechischen Original hin, d. h. hauptsächlich auf Homer, eingeschränkt, ${ }^{39}$ aber hinter der Beurteilung als unbedenkliches Vorgehen bzw. unbedenkliches Versetzen steht wiederum das bereits mehrfach beobachtete Bemängeln einer fehlenden historisch-kulturellen Akkuratesse des Dichters, ohne eine ästhetische Dimension von vornherein geltend $\mathrm{zu}$ machen. ${ }^{40}$ Jedoch ist bei aller Historismus-Kritik $\mathrm{zu}$ betonen, dass die abschließende Bemerkung Krolls auf ein weitaus differenzierteres Verständnis dieser Problematik schließen lässt: Er betont am Ende seines Exkurses zusammenfassend,

Gleichnissen als Beispiele für „,argumenta inter se pugnantia“ angeführt, die daher rührten, dass der Dichter nach der Art und Weise seiner eigenen Zeit spreche - „ex sui temporis ratione“.

38 Kroll 1924, 184. Vor dem Doppelpunkt im Zitat steht ein Fußnotenverweis, in dem er u. a. auf Ebert 1888 verweist. Erwähnenswert ist auch die um einige Jahrzehnte spätere Deutung von Vergils epischem Stil als Anti-Antiquarismus bei Sandbach 1965/66.

39 Problematisch, weil wohl schon mit jeder beliebigen attischen Tragödie relativierbar, ist allerdings der den Exkurs einleitende Satz (Kroll 1924, 178): „Alle Dichtung, die ihren Stoff aus der Heroenzeit nahm, entlehnte die Farben zur Zeichnung des kulturellen Hintergrundes aus dem homerischen Epos.“ Es ist wohlgemerkt von „aller Dichtung“ und den „Farben zur Zeichnung des kulturellen Hintergrundes“ die Rede, was auch bei großzügiger Auslegung des Entlehnens und des kulturellen Hintergrundes konträr zu dem doch sehr apodiktischen Tenor dieser Behauptung ist. S. dazu auch Solodow 1988, 241 (Anm. 19).

40 Auch für Vergil versucht Kroll Abweichungen von Homer z. T. historisch zu verifizieren, um dann doch auch einer künstlerischen Gestaltungsabsicht Geltung zu verschaffen. So sagt er zur deutlich größeren Präsenz an Gold in der „Aeneis“ im Vergleich zu Homer, dass Vergil möglicherweise „von alten Goldschätzen in Italien, wie sie sich namentlich in früheren Gräben, wie dem in Praeneste, finden, Kunde hatte; sicher aber gab den Ausschlag der Wunsch, der Heroenzeit Glanz zu verleihen, ohne daß ängstlich nach der Wahrscheinlichkeit gefragt wurde.“ (Kroll 1924, 179) Die Wahrscheinlichkeit definiert sich hier ähnlich wie schon bei Servius ausschließlich historisch, womit aber nicht nur das aristotelische عikós in dessen Dichtungsdefinition zu absolut ausgelegt wäre. S. o. S. 72, Anm. 246 und Kap. 3.1.3. 
dass die „docti poetae der Alten“ von „einer Pedanterie, wie wir sie von modernen Dichtern gewöhnt sind“441, weit entfernt seien, womit einer solchen Darstellungsweise, die im Kontrast $\mathrm{zu}$ dieser Pedanterie steht, sehr wohl eine gewisse Berechtigung eingeräumt ist.

Eine solche komplexere Sicht zu Aktualisierungen bzw. „Vermischungen von Griechischem und Römischem“ findet sich dagegen schon in Lafayes einflussreicher Abhandlung..$^{42}$ Dessen grundsätzliche Lesart der „Metamorphosen“ könnte, bezogen auf die hier zu diskutierende Thematik, zunächst als traditionell bezeichnet werden, weil er wie Ebert und Haupt das Römische in der mythischen Welt als befremdend ansieht, demgemäß der Leser insbesondere durch Vergleiche dazu angehalten werde, die Illusion einer mythischen Welt zu verlassen, um sich mit der eigenen Gegenwart auseinanderzusetzen: ${ }^{43}$ Historische Abgeschlossenheit und mythische Homogenität werden mit der zeitgenössischen, für den modernen Philologen ebenso historischen Gegenwart des Autors vermengt. Dementsprechend sei es ein Fehler gegenüber der fiktiven Chronologie, dass das sog. heroische Geschehen dem des augusteischen Rom gleiche. ${ }^{44}$ Es ist für Lafaye sogar möglich, Ovids Ungeduld zu erkennen, weil er nicht auf das 14. Buch der „Metamorphosen“, ab dem italische Mythen und italische Geschichte thematisiert werden, warten könne und immer wieder unweigerlich auf Rom $\mathrm{zu}$ sprechen kommen müsse. ${ }^{45}$

Doch ungeachtet dieser Ausdrucksweise bemüht sich Lafaye anders als die zuvor genannten Philologen um eine weiterreichende Erörterung dieses Befundes anhand ähnlicher Beispiele bei Kallimachos. Dass dieser so dichte, obwohl er ein unbestrittener Kenner vergangener Ereignisse sei, ist Anlass für folgende scharfsinnigen Bemerkungen:

Est-elle beaucoup plus hardie qu'une foule d'autres, sans lesquelles l'art n'existerait pas? Y a-t-il même jamais eu, dans aucun temps, un poète qui ait pu s'en affranchir complètement, et alors dans quelle mesure est-elle acceptable? Il faudrait ignorer combien la question est délicate pour reprocher sévèrement à Ovide d'avoir mêlé l'antique et le moderne et pour ne pas goûter nousmêmes, comme ses premiers lecteurs, la saveur particulière de ce mélange. ${ }^{46}$

41 Ebd., 184. Mit „modernen Dichtern“ werden am ehesten Vertreter des poetischen Realismus gemeint sein, die in der letzten Hälfte des 19. Jahrhunderts und bis ins 20. Jahrhundert hinein in bürgerlich-konservativen Kreisen stark rezipiert wurden. Zu einer tatsächlich modern zu nennenden Ästhetik zu dieser Zeit s.u. Kap. 7.2.2.2.

42 Vgl. die Einleitung von Albrechts zum Nachdruck des Lafaye-Textes (von Albrecht 1971, V*).

43 Vgl. Lafaye 1904, 113 f.: „,nous avons un peu de peine ensuite à remonter le cours des siècles pour revenir à Lycaon ou à Cadmus.“

44 Vgl. ebd., 110.

45 Vgl. ebd., 113: „Il n'a pas la patience d'attendre le quatorzième chant; il faut qu'il les revoie de temps en temps comme si l'Olympe même était pour lui un lieu d'exil.“ Zu einer treffenden Bewertung dieser Sichtweise s. Tronchet 1998, 361.

46 Lafaye 1904, 114. 
Die von ihm als „,anachronisme des idées et du langage“ ${ }^{\text {“47 }}$ bezeichnete Aktualisierung steht so gesehen in einer Reihe von Darstellungstechniken, die in jeder Kunst Anwendung finden und zudem einen so essentiellen Rang in ihr einnehmen, dass sie zeitunabhängig und unvermeidbar sind. ${ }^{48}$ Daran wird deutlich, dass ein bloßes Nachweisen, wie es Ebert explizit als seine Zielsetzung formuliert, eigentlich nur der vorbereitende Schritt dafür ist, jene Angemessenheit bzw. jenen Grad des Akzeptierbaren und, wodurch er festgelegt ist, zu ergründen, sodass der besondere Effekt dieser Mischung, die „saveur particulière de ce mélange“, benannt werden kann. ${ }^{49}$

Was die tatsächlichen Anachronismen betrifft - Lafaye spricht von den „sogenannten Anachronismen“50 - besteht kein Zweifel an ihrer Fehlerhaftigkeit. Beachtenswert ist dabei allerdings, dass auch diese Fehler mit einem ganzen Bündel an Hypothesen zu erklären versucht werden: mit der von Ovid selbst andernorts angesprochenen Fehlerhaftigkeit und Unabgeschlossenheit der „Metamorphosen“ ,51 mit dem innertextlichen Abstand der widersprüchlichen Elemente, mit der Vielzahl verschiedener Quellen, mit der großen Anzahl an Figuren und Genealogien, mit der fehlenden Achtsamkeit des Autors, mit dessen durch die alexandrinischen Dichter erwecktem Interesse, höchst kunstvolle Arrangements möglichst einfach aussehen zu lassen, und zuletzt mit dessen Erinnerungsgabe, die stark ausgeprägt und von seltener Schnelligkeit ist. ${ }^{52}$ Angesichts einer solchen Vielzahl an Gründen erscheint es Lafaye konsequenterweise ganz im Gegenteil verwunderlich, dass es nicht noch viel mehr derartige Widersprüche gibt. ${ }^{53}$

Das Quantitätsargument, das bereits bei den anderen besprochenen Abhandlungen in verschiedener Ausprägung beobachtet worden ist, wird hier sogar positiv umgedeutet, um die Brillanz des Dichters zu unterstreichen, der trotz dieser offenbar beträchtlichen Schwierigkeiten ein lesbares Gedicht zu Papier gebracht habe.

47 Ebd.

48 Wiederum in Analogie zu Goethes Diktum, „daß alle Poesie eigentlich in Anachronismen verkehre“ (s. o. S. 28f., Anm. 120).

49 Der Hinweis, dass man die eigentliche Problematik nicht ignorieren dürfe, indem man Ovid aus dem eigenen ästhetischen Empfinden heraus heftig kritisiert, offenbart außerdem, dass schon zu Beginn des 20. Jahrhunderts wahrgenommen wurde, dass methodisch verallgemeinernde Deutungen eher der Legitimation des eigenen Standpunkts im Untersuchungsobjekt zu dienen scheinen.

50 Vgl. ebd., 81: „On a relevé dans les Métamorphoses ce qu’on appelle des anachronismes; entendons par là des fautes qui troublent la suite hypothétique établie par Ovide lui-même entre les fables. “ Die tatsächlich von einem Erzähler dargebrachte Welt gilt als „hypothétique“, während die Wissensebene des Rezipienten bzw. Interpreten als faktisch angenommen wird. An späterer Stelle spricht er auch explizit von einem Fehler (pèche) gegen die „chronologie réelle des temps historiques“ (ebd., 110). 51 Vgl. dazu die einschlägigen Stellen in trist. I, 7. Zur Deutung dieser und anderer Aussagen Ovids zu seinen „Metamorphosen“ s. die nach wie vor überzeugenden Bemerkungen von Kraus 2 1982, 120 - 122. Mit direktem Bezug zum Anachronismus vgl. Tronchet 1998, 360.

52 Vgl. Lafaye 1904, $81 \mathrm{f}$.

53 Ebd., 81. Noch z. B. Cole 2004, 414 (Anm. 142), beruft sich auf diese Argumentation als „Lafaye’s verdict“. Zu Cole s.u. Kap. 4.1.2. 
Die Schwächen dieser fraglos zugespitzten Position treten aber allein dadurch zu Tage, dass der eigentliche Befund für die Behauptung, dass sich dieses Phänomen oft oder selten niederschlägt, so evident zu sein scheint, dass er gar nicht bewiesen werden muss, z.B. durch eine konkrete, numerisch nachvollziehbare Auflistung. ${ }^{54}$ Und abgesehen von dem fehlenden quantitativen Textzeugnis, auf das sich eine These tatsächlich stützen könnte, ist es noch gravierender, dass die Festlegung, ab wann diese Auffälligkeit im Text durch ihre bloße zahlenmäßige Manifestation signifikant ist, vollkommen beliebig ist. ${ }^{55}$ Auch die kolportierte Menge an Stoffen und Genealogien, gemessen an denen der Anachronismus in zu vernachlässigender Häufigkeit vorkommt, entzieht sich einem objektivierbaren Urteil.

Folglich wird ein ungenau erfasstes und beschriebenes Phänomen mit einem ungenauen, weil willkürlichen Maßstab aufgrund eines ungenauen, weil subjektiven Vergleichspunktes als nicht signifikant bewertet, damit dieser Befund - das steht womöglich zuvorderst in dieser Ursachenkette - im eigenen hermeneutisch-historistischen Weltbild seinen ihm zugehörigen Platz finden kann. ${ }^{56}$

\subsubsection{Beginn einer „objektivere[n] Würdigung“ - Arbeiten ab den 1930er Jahren}

Eine wesentliche Änderung in der wissenschaftlichen Beschäftigung mit Anachronismus und Aktualisierung in den „Metamorphosen“ vollzieht sich parallel zur allgemein günstigeren bzw. objektiveren ${ }^{57}$ Beurteilung Ovids, die schon bei Lafaye in Ansätzen erkennbar und in den nachfolgenden Jahrzehnten von wegweisenden neuen Impulsen geprägt worden ist. ${ }^{58}$

54 Lafaye führt selbst lediglich ein einziges Beispiel (Atlas) an und nicht einmal das vollständig, weil er nur zwei Stellen, eine aus dem zweiten und eine aus dem vierten Buch, nennt. Vgl. Lafaye 1904, 81. Ähnlich unbefriedigend ist die Erklärung Grimals, mit der er zugleich Lafayes Einwand kommentiert, beim Anachronismus der Atlas-Figur: Einerseits will er zwei verschiedene Atlas-Figuren ausmachen, um letztlich doch auch die Inkonsistenz zu erkennen, die vielleicht bei einer nochmaligen Durchsicht hätte vereinheitlicht werden können. Vgl. Grimal 1958, 247 f. Zur Kritik an beiden vgl. Tronchet 1998, 372. $\mathrm{Zu}$ Atlas s.u. Kap. 5.1.2.4.

55 Ab wann nämlich, so ließe sich in Anlehnung an den sorites fragen, wird der Haufen zum Haufen und wie kann das überhaupt ermittelt werden, wenn er nicht einmal quantitativ beschrieben wird? 56 Die erste oder eine der ersten Abhandlungen, die eine Absicht in den von Anachronismen erzeugten Unterbrechungen der Chronologie sieht, ist wohl Crump 1931, 200, was im Allgemeinen mit den nachfolgend beschriebenen Tendenzen übereinzustimmen scheint.

57 Das Zitat im Titel stammt aus Buchheit 1966, 81, und ist dort bezogen auf die „Beurteilung der Komposition“ der „Metamorphosen“.

58 Vgl. ebd., 80-82, mit zahlreichen bibliographischen Angaben. Ebenso interessant ist die etwas ausführlichere Diskussion bei Doblhofer 1960, 63-69. Erinnert sei stellvertretend an Heinzes 1919 und Dillers 1934 erschienene Abhandlungen. 
Diese Entwicklung spiegelt sich bereits sehr nachhaltig in Walther Kraus' REArtikel zu Ovid wider, der 1942 in der Enzyklopädie veröffentlicht wurde und als erster, äußerst wichtiger Beitrag an dieser Stelle vorgestellt werden soll. ${ }^{59}$

Das in den früheren Arbeiten absolut scheinende Prinzip der mythischen Geschichtlichkeit und die oft postulierte Abgeschlossenheit einer „heroischen Zeit“ tritt bei Kraus angesichts der Erkenntnis zurück, dass Ovids Streben nach Veranschaulichung und geistig-pointierter Durchdringung seines Materials wenig Sinn „[f]ür die geheimnisvolle geschichtliche Wirklichkeit des Mythos“600 erkennen lässt. Unter Betonung der rhetorischen Schulung Ovids, durch die er das in einem Stoff liegende Potenzial erkennen und auf seine Wirkung hin bestmöglich künstlerisch zum Vorschein bringen konnte, ziehe er durch die Wahl der Geschichten einerseits und durch die spezifische Art der Darbietung andererseits die Sagen „in die Sphäre des Nahen, unmittelbar Verständlichen. Er modernisiert und verbürgerlicht bis an die Grenzen der Travestie. “61

Dass dieses Verfahren nicht als Anachronismus, sondern als Modernisierung und Verbürgerlichung bezeichnet wird - von ihrem Prinzip her mit der Aktualisierung gleichzusetzen $-{ }^{62}$ zeigt sehr deutlich die Verschiebung von einem hauptsächlich historischen Standpunkt hin zu einem Versuch, die beobachteten Eigenarten aus einer primär ästhetischen Perspektive zu beschreiben und somit deren Wert als etwas Eigenständiges anzuerkennen. ${ }^{63}$ Formal bedingt ist die Beschreibung auf Prinzipielles und nur wenige, besonders illustrative Beispiele beschränkt, darunter auch der locus classicus in met. I, 169-176, der in nahezu jeder Arbeit zu dieser Thematik diskutiert wird. ${ }^{64}$

Weiterhin zu erwähnen ist Kraus’ treffender Kommentar, der sich an die Beobachtung anschließt, dass in den „Metamorphosen“ noch im Absurdesten auf die Folgerichtigkeit der entsprechenden Wirkung geachtet wird:

59 Die 1968 in überarbeiteter Fassung im WdF-Band zu Ovid neu abgedruckte und unveränderte zweite Auflage wird hier zitiert.

60 Kraus $^{2}$ 1982, 117. Er verweist hierbei interessanterweise auf just solche Stellen in Ovids Euvre (nicht jedoch auf am. III, 12), in denen die Unglaubwürdigkeit des Mythos unterstrichen wird. Weiteres dazu findet sich oben in Kap. 3.1.2.

61 Ebd.

62 S. o. Kap. 3.3.3.1.

63 Aus den Beispielen, die Kraus gibt (vgl. ebd., 117 f.), wird auch noch einmal deutlich, wie wenig sich zur Beschreibung dieser Stellen die Bezeichnung „Anachronismus“ eignen würde, weil die hierin vorausgesetzte Zeitlichkeit meist nachrangig oder gar nicht vorhanden ist, z. B. bei einem Flussgott, der als Vater zu seiner Tochter spricht, oder bei Kirkes mondänen Allüren.

64 Einzelne Titel anzuführen wäre daher ein uferloses Unterfangen. Erwähnenswert ist, dass schon Raffaele Regio auf diesen Aspekt hinweist („,videtur autem Ovidius alludere ad Palatium Romanum: in quo imperatores cum principibus habitabant“ - ,Es scheint aber Ovid auf den römischen Palatin anzuspielen: Auf diesem wohnten die Kaiser zusammen mit der führenden Gesellschaftsschicht “ Regius 1517, 6 (ad met. I, 173)). Vgl. für weitere Angaben die m.W. jüngste Monographie dazu Lenzi 2015. 
[W]enig ernst ist es ihm mit Realität und Konsequenz, sofern sie außerhalb des unmittelbar poetisch Wirksamen liegen. Die Geographie ist ihm ebenso gleichgültig [...] wie die Chronologie; ${ }^{65}$

Sowohl die Betonung des künstlerischen Prinzips als auch das Absetzen der poetischen Wirksamkeit gegenüber einer außertextlichen Wirklichkeit, womit de facto obwohl es nicht als solches geäußert wird - auf die Gestaltungsmöglichkeiten hingewiesen wird, die in einem fiktionalen Umfeld im Gegensatz zu einer historisch festgelegten Realität stehen, sind deutliche Anzeichen für die philologische Aufwertung des ovidischen Euvres. Dessen beabsichtigte Priorisierung des Poetischen wird sogar bei wohl einem der strittigsten Fälle der „Metamorphosen“ verteidigt, der auch heute noch Anlass gibt, wegen des Anachronismus von einer Interpolation auszugehen. ${ }^{66}$

Die Anachronismen jedoch - das macht der beiläufige Hinweis auf die als „Flüchtigkeit“ erklärten Widersprüche deutlich, die, wie Kraus notiert, in der Ausgabe von Haupt/Ehwald erwähnt sind $-^{67}$ fallen hier explizit nicht darunter, so wie auch das Denken bzw. Erwarten von historischer Richtigkeit in gewisser Weise insofern zum Tragen kommt, als in der stofflichen Anordnung „gewaltsam [...] angeknüpft“ und „ein gewaltsamer Übergang“68 ausgemacht wird. Die von ihm bei Ovid beschriebene Gleichgültigkeit gegenüber der Chronologie ist allerdings nicht mit letzter Konsequenz als die Absage an ein implizit vorhandenes Ordnungsprinzip zu verstehen, sondern als dessen innovative Modifizierung und Relativierung, sodass auch dies ganz dem ausgewogenen Tenor des Artikels entspricht. ${ }^{69}$

Von den in der Folge dieses Übersichtsartikels publizierten Interpretationen ist hier als erste der 1966 erschienene Aufsatz von Buchheit zu „Mythos und Geschichte“ in den „Metamorphosen“ zu nennen, der sowohl die bis zu dieser Zeit erfolgte Entwicklung in der Ovid-Forschung überblicksweise nachzeichnet und reflektiert als

65 Kraus ${ }^{2} 1982,120$. Allerdings ist es leicht widersprüchlich, wenn Kraus wenige Seiten respektive Spalten vorher noch davon spricht, dass Ovid es unternahm, „[d]ie Masse des [im RE-Artikel jedoch richtig: der] Metamorphosen chronologisch zu ordnen“ (S. 109) und „das Prinzip des durchgängigen historisch-chronologischen Zusammenhangs durchzuführen“ (S. 111).

66 Die Rede ist von Pythagoras' Verweis in met. XV, 426-430 auf den Niedergang von u.a. Sparta, Mykene und Athen, obwohl besonders letztere Stadt sich zur Zeit des historischen Pythagoras - im 6. Jh. v. Chr. - allmählich erst überhaupt zu einer überregionalen Macht entwickelte. Zur Diskussion der Stelle s. Tarrant 2000, 433-435. Hierzu Kraus’ abschließendes Urteil: „Derlei ist natürlich nicht als Flüchtigkeit anzusehen [...]; es ist kennzeichnend für die Verwegenheit, mit der O. alles dem Gesichtspunkt der rhetorischen Wirkung unterordnet, deren er sich sicher weiß“ (Kraus ${ }^{2} 1982,120$ ). S. auch u. S. 161, Anm. 184.

67 Vgl. ebd.

68 Beide Zitate ebd., 111.

69 Natürlich kann ein Text diesen Eindruck eines gewissen geographischen und temporalen Desinteresses nur aus einem ihm prinzipiell zugestandenen oder von ihm erwarteten Ordnungsprinzip entfalten - und zwar dort, wo dies angesichts des Bedeutungsgehalts der fiktiven Objekte möglich ist. Vor diesem Hintergrund lässt sich der oben festgestellte Widerspruch in Kraus’ Urteil (s. o. S. 100, Anm. 65) problemlos auflösen. S. dazu Wheeler 2002, 181. 
auch eine motivspezifische Erörterung der Funktion zeitgeschichtlicher Elemente in der Welt der „Metamorphosen“ gibt. $^{70}$

Dabei wird die bei Kraus eingeschlagene Denkrichtung wiederaufgegriffen, wenn die Darstellungsabsicht Ovids als eine in der Evolution vom Chaos zum Kosmos stattfindende Antizipation des augusteischen Roms gewertet wird. ${ }^{71}$ Gleichwohl aber offenbart die Zielsetzung, zeitgeschichtliche Anspielungen und die Parallelisierung von Augustus und Jupiter nachwiesen zu wollen, ähnlich wie bei Eberts „Beweisführung“ ein methodisches Problem, das einer grundlegenderen Beschreibung der Aktualisierungen eher hinderlich ist.

Denn dem Phänomen selbst wird vor allem dann Signifikanz beigemessen, wenn es der Argumentation dient, d. h. wenn sich daran zeigen lässt, dass mit der Repräsentation zeitgenössischer Objekte in der Welt des Mythos der politische Zustand zur Abfassungszeit des Gedichts vorweggenommen wird. Das genaue Funktionieren dieses Verweises auf der sprachlichen Ebene, ob dies als Gleichnis, Metapher, in Form eines Erzähler- oder Figurenkommentars etc. geschieht und mit welcher Deutlichkeit dies aus seinem narrativen Kontext hervorgeht, spielt hierfür eine untergeordnete Rolle. ${ }^{72}$ Und die Wahl der Beispiele bzw. des einen Beispiels muss als selektiv und unvollständig erscheinen, wenn aus der mit nicht wenig Aufwand von Ovid beschriebenen Szenerie in met. I, 168-176 lediglich der Verweis auf die Palatia caeli herausgegriffen wird. ${ }^{73}$ Aus methodischer Sicht ist außerdem problematisch, dass Aktualisierungen, noch bevor sie eingehender beschrieben werden, bereits nach ihrer Funktion interpretiert sind, sodass gleichzeitig impliziert ist, dass jedes Beispiel in den „Metamorphosen“ dieser dichterischen Intention entspricht. ${ }^{74} \mathrm{Ob}$ dies aber in

70 Des Weiteren sei der Artikel von Buchheit wegen seiner ausgewogenen Themenstellung auch stellvertretend für andere zeitgleiche oder frühere Arbeiten betrachtet, die die von ihm diskutierten Stellen schon vorher in ähnlicher Weise besprechen (Doblhofer 1960; von Albrecht 1963 und Ludwig 1965).

71 Vgl. Buchheit 1966, 84f. Das bei Kraus eher allgemein beschriebene Prinzip der effektvollen Inszenierung ist hier somit auf ein Motiv hin konkretisiert.

72 So ist die Aussage ungenau, „daß Ovid ausdrücklich die Himmelsburg Jupiters als Palatia caeli bezeichnet“ (ebd., 85), da weder von einer Himmelsburg die Rede ist noch diese „ausdrücklich“ als Palatia caeli (was sehr wahrscheinlich den Hügel als Ganzen meint und nicht den Palast; s.u. S. 236, Anm. 99) bezeichnet wird - das würde den beträchtlichen Aufwand Ovids, die Aussage abzumildern und beide Ebenen gleichnishaft nebeneinander zu stellen, gänzlich unbeachtet lassen. Die Unterscheidung unterbleibt auch bei Ludwig 1965, 18; anders dagegen von Albrecht 1963, 51, der richtungsweisend von einer „kühne[n] Metapher“ spricht. S. dazu u. Kap. 7.1.3.

73 In z. T. sehr deutlicher Anlehnung an Kraus werden daneben aber bei Doblhofer 1960, 71 weitere Beispiele genannt; ebenso ist bei von Albrecht 1963, 51 neben den Palatia caeli auf einen weiteren Aspekt verwiesen.

74 S. dazu auch die Bemerkungen in der Einleitung (Kap. 1.3). Weiterhin ist hier noch die - an dieser Stelle nicht genauer nachzuzeichnende - Diskussion über Ovids Humor und Ironie relevant, d. h. wie affirmativ (als „Huldigung“, Ludwig 1965, 18) oder humoristisch (s. dazu Wilkinson 1955, 195f., Doblhofer 1960, 71 und den Hengst 1988, 355) die Darstellung in met. I, 168-176 zu verstehen ist. Zur Ironie in den „Metamorphosen“ allgemein s. Krupp 2009. 
anderen Passagen, in denen die diegetische Umgebung die politische Parallele nicht so deutlich evoziert, und bei vorwiegend hintergründigen Ausstattungsmerkmalen immer in derselben Weise zum Tragen kommt, ist fraglich.

Ungeachtet dieser Kritik im Detail sind neben der ausführlichen quellenkritischen Verortung einzelner Motive, von der aus die Anders- oder Neuartigkeit von Ovids Erzählung sichtbar wird, die zusammenfassenden Bemerkungen Buchheits aufschlussreich. Darin wird der „eigentümliche Schwebezustand zwischen Mythos und Geschichte“ betont, der das Gedicht vor einer oberflächlichen Betrachtung und davor, den einzelnen Mythos lediglich „,in seiner ,realen“ Aussage“ verstehen zu wollen, bewahre. ${ }^{75}$ Und dadurch unterscheide sich auch Ovid von seinen Vorgängern, besonders von Vergil, deren bzw. dessen Mythenbilder als Beitrag an einem römischen Geschichtsnarrativ verstanden werden können, während die „Metamorphosen“ zwar ebenso auch aus einer durch und durch römischen Perspektive erzählt werden, jedoch als „Deutung des Lebens insgesamt [...] eine Wirkung unabhängig von zeitlich gebundener Aussage“76 entfalten. Statt Bedenken- und Respektlosigkeit gegenüber der historischen Wahrheit findet nicht nur das Oszillieren Ovids zwischen mythischer und geschichtlicher Realität als originelle Weiterentwicklung bereits bestehender Motive Anerkennung, es wird noch dazu einer vereinfachenden Lesart deutlich entgegengesetzt.

Als ein Aufgreifen und Fortführen dieses Ansatzes können Bernbecks 1967 erschienene „Beobachtungen zur Darstellungsart in Ovids Metamorphosen“ angesehen werden, in denen die Ambiguität in der so plausiblen, aber zugleich unwahrscheinlichen Erzählweise Ovids untersucht wird. Der Stil selbst ist darin Gegenstand der Analyse, wobei die Beschreibung von Aktualisierungen oder Anachronismen im Allgemeinen und Exemplarischen bleibt. ${ }^{77}$ Mag auch die Diskussion daher im Detail hilfreich sein, können die Ergebnisse für eine kategorische Erschließung nur bedingt Anknüpfungspunkte bieten, wenn Aktualisierungen als „überraschende Verzerrungen der traditionellen Vorstellungen“ von der „Vermischung zweier Bildbereiche“78 bis hin zu Paradoxien und den „zahlreichen Inkonsequenzen“79 reichen. Neben der

75 Beide Zitate Buchheit 1966, 107.

76 Ebd., 108. S. auch o. Kap. 3.1.2.

77 S. dazu besonders das dritte Kapitel „Inhaltliche Besonderheiten der ovidischen Vorstellungen“ (Bernbeck 1967, 80 -116). Aussagen wie „Er nimmt keine Rücksicht darauf, ob diese Erfindungen den Erfordernissen der Erzählung entsprechen. Im Gegenteil: er wählt bewußt solche Vorstellungen, die in fremder Umgebung zu inneren Unstimmigkeiten führen“ (S. 91) erinnern zudem sehr an die Bewertung der „Metamorphosen“ früherer Jahrzehnte, nur mit umgekehrtem Vorzeichen (s. hierzu auch die Einschätzung bei von Albrecht 1981, 2330). Denn was die „Erfordernisse der Erzählung“, „die fremde Umgebung“ und „die inneren Unstimmigkeiten“ sind, bedarf als interpretatorische Prämisse offenbar nicht der Explikation, mag das Verfahren selbst auch als absichtliche Bearbeitung des Dichters aufgefasst werden.

78 Bernbeck 1967, 92.

79 Ebd., 97 (Anm. 31). Ähnlich lautet die abschließende Beurteilung in der Rezension von Kenney 1968. Es ist aber nicht ersichtlich - womöglich auch, weil kein Grund dafür angeführt wird -, warum 
Beschränkung auf wenige, besonders auffällige und überwiegend aus den ersten Büchern stammende Textstellen und einer wohl zu einseitigen Übergewichtung des Formalen gegenüber dem Inhaltlichen in dieser Abhandlung ${ }^{80}$ dürfte sich insgesamt durch die „Beobachtungen“ kein klarerer Blick auf diese Thematik ergeben. ${ }^{81}$

Grundlegend anders ist dies bei von Albrechts 1981 erschienenem Aufsatz zu „Mythos und römischer Realität“. Dass dieser nach wie vor als einer der wichtigsten Beiträge $\mathrm{zu}$ werten ist, liegt daran, dass der interpretatorische Standpunkt dezidiert über jene historistische Perspektive aus früheren Zeiten, aber auch über die ab der Mitte des 20. Jh. an Einfluss gewinnende Einschätzung, Ovids Darstellung gleiche einem manierierten, möglichst effektvollen Spiel, hinausgehen will; ${ }^{82}$ und letztlich daran, dass die Einbettung der an mehreren Beispielen illustrierten Beobachtungen in eine ästhetischen Gesamtbewertung der „Metamorphosen“ unter Berücksichtigung der hierfür relevanten literarischen Traditionen mündet. ${ }^{83}$

Ovid gehe es, so von Albrecht, in erster Linie darum, dem religiös weitgehend verblassten und deswegen sehr freizügig dichterisch bearbeitbaren Mythos neue Vitalität und Aussagekraft zu verleihen und ihn somit in seiner ursprünglichen Funktion zu reaktivieren, „Wege zur Deutung der Natur und des mit ihr verflochtenen menschlichen Schicksals zu erschließen."84 Dieses Verfahren finde einen deutlichen Niederschlag in epischen Gleichnissen, denen per se die Eigenschaft zu Teil ist, die mythische Welt der Erfahrungswelt des zeitgenössischen Publikums gegenüberzustellen, des Weiteren in sog. „historischen Anspielungen“ sowie in der fein ausdifferenzierenden psychologischen Zeichnung der Figuren. ${ }^{85}$ In diesem Sinn sei auch das Paradoxe und Absurde kein spielerischer Selbstzweck, sondern die Möglichkeit, die

nach Kenney „Military imagery“ nicht „in the class of anachronistic contemporary elements in the narrative“ (ebd., 58) gehört. Vgl. dagegen von Albrecht 1981, 2336.

80 Vgl. auch Kenney 1968, 58 f.

81 So liefert beispielsweise die Besprechung von met. I, 168-176 in dieser Hinsicht wenig Neues, auch wenn sie ausführlicher ist und genauer auf die einzelnen Motive dieser Szenerie eingeht. Vgl. Bernbeck 1967, $92 \mathrm{f}$.

Im selben Zug kann auch die Überblicksarbeit von Galinsky genannt werden, weil sie sich bei der Besprechung der „incongruity of ideas“ (Galinsky 1975, 28) auf Bernbeck bezieht und Ovid an anderer Stelle eine typische „preference for anachronism“ (ebd., 235) zuerkennt, wobei wie bei der „incongruity“ eine Aktualisierung gemeint ist. Ebenso werden z. T. Anachronismen als „deliberate exceptions to the chronological sequence“ (ebd., 85) erwähnt, allerdings sind dabei auch Anachronien als Beispiele aufgeführt. Dass letztere definitionsgemäß keine Anachronismen sein können, ist an anderer Stelle schon erwähnt worden. S. o. Kap. 3.2.2.1.

82 Vgl.von Albrecht 1981, 2330 f. Bemerkenswerterweise strebt von Albrecht ähnlich wie Buchheit, der für Ovid im Allgemeinen jene „objektivere Würdigung“ (s. o. S.98, Anm. 57) feststellt, „eine objektivere Standortbestimmung“ (ebd., 2331) zu diesem Thema an.

83 Vgl. ebd., bes. $2338-2340$.

84 Ebd., 2342. Ganz analog zu den Beobachtungen in Kap. 3.1.2.

85 Allerdings ist das, was als Beispiel sowohl für die „historische Anspielung“ als auch für die „Psychologie“ angeführt wird, sehr großzügig aufgefasst, d. h. nicht auf einer klaren Unterscheidung basierend. 
menschliche Erfahrungswelt bis an Grenzsituationen und Schwebezustände heranzuführen. ${ }^{86}$

So überzeugend die Deutung für das Gesamtgedicht ausgehend von den Einzelbefunden einerseits demnach ist, scheint der allgemeine Zugriff Raum für eine grundsätzliche und systematische Erfassung offen zu lassen bzw. den Weg dorthin sogar selbst zu weisen. ${ }^{87}$ Unter Hinzuziehen einer noch definitiveren terminologischen Unterscheidung zwischen Anachronismen und Aktualisierungen ${ }^{88}$ kann diese Sachund Sprachanalyse auf ein valideres Fundament gestellt und erneut bewertet werden. ${ }^{89}$ Dass es sich dabei weitestgehend um eine Ergänzung und Ausweitung der in diesem Artikel dargelegten Deutung der Aktualisierungen handelt, spricht sehr für die Plausibilität der Argumentation. ${ }^{90}$

Für die tatsächlichen Anachronismen bietet Solodows 1988 erschienene Gesamtdeutung der „Metamorphosen“ neben einer weiteren hilfreichen Besprechung von Aktualisierungen neue bzw. weitere Impulse.

Ausgehend von der Beobachtung, dass Ovid zum einen auf unterschiedliche und unterschiedlich markante Weise Struktur- und Ordnungsprinzipien evoziert, diese aber zum anderen immer wieder als unvollständig oder unbeachtet erscheinen lässt, werden Anachronismen als Inkonsistenzen in der Chronologie erwähnt. ${ }^{91}$ So ermög-

86 Vgl. ebd., $2337 \mathrm{f}$.

87 Vgl. ebd., 2331. Sowohl über die Reflexion zur Rolle des Mythos in den „Metamorphosen“ als auch über die sachliche und sprachliche Analyse der erzählten Welt jenes Gedichts sei dies auf eine objektive Weise zu bewerkstelligen.

88 So wird zunächst auf die Bewertung des Phänomens bei Haupt/Ehwald als „Anachronismen“ und „Geschmacklosigkeiten“ kontrastiv hingewiesen (ebd., 2330), dann aber selbst von „sogenannten Anachronismen“ (2336) und im Vergleich mit den Renaissance-Malern nur mehr von „Anachronismen“ (2341) gesprochen; des Weiteren werden eher funktionale Umschreibungen wie „historische Anspielungen“ (2335), „vergegenwärtigen“ (2337) und auch „aktualisieren“ (2341) gebraucht, mit denen z. T. auch andere Erscheinungen beschrieben werden können. S. dazu auch den ähnlichen Sprachgebrauch bei von Albrecht 1979, 133 und von Albrecht 2008, 227.

89 Auch die Stellung der einzelnen Gleichnisse und den sprachlogischen Unterschied zur Aktualisierung könnten so ausgehend von diesen und anderen Beiträgen noch eingehender untersucht werden. S. grundsätzlich zu Aktualisierungen und Gleichnissen Kap. 7.1. Die jüngste Abhandlung zu dieser Problematik, Lücht 2019, bietet jedoch trotz manch hilfreicher Systematisierung - die aber nicht einmal alle Beispiele umfasst (ein flüchtiger Blick erkennt, dass met. V, 389 fehlt), wodurch auch die dargebotene Statistik auf fraglichem Grund steht - einen konventionellen hermeneutischen Standpunkt mit all seinen methodischen Nachteilen. Folglich wird bei der Analyse der Gleichnisse weder eingehender die Stellung der Erzähler beachtet, obwohl dies nicht irrelevant ist (s.u. Kap. 5.2.2), noch eine präzise Definition zu „Anachronismen“ (Lücht 2019, 116) oder eine grundsätzliche Problematisierung gegeben, inwiefern Gleichnisse anachronistisch sein können. Wie wenig zufriedenstellend die Beobachtungen dann sein müssen, zeigt die Besprechung des Rohrbruch-Gleichnisses S. 115-118.

90 Einen Eindruck davon vermittelt auch der von von Albrecht angestellte Vergleich von Ovid, der die vornehmlich griechischen Mythen für ein römisches Publikum aktualisiert, mit Odysseus, „der den Schatten der Unterwelt durch lebendiges Blut neues Leben verleiht“ (von Albrecht 1981, 2337 und von Albrecht 1976, 290).

91 Vgl. Solodow 1988, 25 und 29. Ähnlich bereits Otis ${ }^{2} 1970,373$. 
licht Solodow nicht nur eine ausgewogenere Betrachtung dieser scheinbar störenden, unharmonischen Elemente, sondern er eröffnet in der Folge auch die Möglichkeit, darin funktionale Aspekte in Erwägung zu ziehen..$^{92}$ Die von ihm untersuchten Beispiele - insgesamt weder gering an Zahl noch unauffällig - ${ }^{93}$ dienten so dem Zweck, dass das leserseitige Gefühl einer zeitlichen Vorwärtsbewegung in der Erzählung abgestumpft bzw. getrübt wird. ${ }^{94}$ Verglichen jedoch mit anderen Erzähltechniken, vor allem den zahlreichen Analepsen, sei dieser trübende Effekt eher gering. ${ }^{95}$

Beachtenswert ist weiterhin Solodows Besprechung von Formen der Aktualisierung, die unter den Schlagwörtern „Anachronism“, „Romanization“ und „Modernization“ und im größeren Kontext der Auseinandersetzung mit der Mythologie der „Metamorphosen“ erfolgt. $^{96}$ Dabei sind sowohl die einleitenden methodischen Überlegungen wegweisend, in denen der Frage nachgegangen wird, was als Anachronismus in Betracht zu ziehen ist und welche interpretatorischen Probleme damit verbunden sind; ${ }^{97}$ als auch hilft die Vielzahl an Passagen, die einzeln vorgestellt und in ihrer Besonderheit durch Beispiele bei Homer und Vergil kontrastiert werden, einen genaueren Eindruck von diesem Phänomen zu gewinnen.

Die Frage, ob es einem zwar literarisch gebildeten, aber nicht mit philologischer Akribie lesenden Rezipienten möglich gewesen sei, jedes Ausstattungsdetail nach seiner Konformität gegenüber der epischen Tradition hin zu betrachten und die Signifikanz etwaiger Unterschiede zu erkennen, ${ }^{98}$ berührt ein nicht unerhebliches Problem. ${ }^{99}$ Dass man außerdem bei den Gleichnissen darauf achten müsse, von welcher Erzählinstanz aus diese erzählt werden, weil im Falle intradiegetischer Erzähler die Bildebene des Gleichnisses ebenso der diegetischen Welt angehören müsste, rückt ein bis dahin weitgehend unbeachtetes Problem ins Bewusstsein. ${ }^{100}$

92 Vgl. auch die Bemerkung zu einem Anachronismus von Anderson 1972, ad met. VI, 414-420: „Ovid does not commit this ,mistake“ accidentally; he is toying with his audience.“

93 Zur Problematisierung des Quantitätsarguments s. das vorherige Kapitel. Vgl. auch Rosati 2002, 280, der mit explizitem Verweis auf Galinsky und Solodow von „numerous anachronisms“ spricht ohne dies, wie jene selbst, tatsächlich dann an „zahlreichen“ Beispielen, gewissermaßen „numerisch“ $\mathrm{zu}$ erhärten.

94 Vgl. Solodow 1988, 29.

95 Vgl. ebd.

96 Interessanterweise werden hierfür weder die oben vorgestellten Arbeiten Eberts noch die von Albrechts berücksichtigt.

97 Ganz grundsätzlich wird das schon an Solodows einleitender Feststellung sichtbar, dass für Ovid der Mythos allen voran literarischer Stoff sei, sodass in den „Metamorphosen“ Mythologie als ein Synonym zu Fiktion bezeichnet werden könne. Vgl. ebd., 75. Ähnlich den Schlussfolgerungen in Kap. 3.1.2.

98 Vgl. ebd., $76 \mathrm{f}$.

99 S. dazu unten die Überlegungen in Kap. 7.1.3.3.

100 Vgl. ebd., 76. Zur Hypodiegese s. o. S. 52f. und u. Kap. 5.2. Wie bereits erwähnt, wiederholt noch von Albrecht 1981, 2332, dass im Normalfall bei einem Gleichnis kein Anachronismus auf der Bildebene vorliegen kann. 
Unter Betonung sowohl der Intensität ${ }^{101}$ als auch der Häufigkeit ${ }^{102}$ dieser zeitgenössischen Realien sei dabei nicht nur eine funktionale Ähnlichkeit mit anderen Erzählweisen erkennbar, die den Mythos wie auch die Instanz, die den Mythos vermittelt, als zeitgenössisch und als dem Rezipienten zugänglich ausweisen, sondern auch eine grundsätzliche Andersartigkeit zur Verwendung bei Vergil: Bei diesem diene dies dazu, das Bild einer glänzenden, ehrwürdigen Vergangenheit vorzustellen oder einen gegenwärtigen Zustand in Aeneas' Zeit zu präfigurieren und durch seinen mythischen Ursprung zu legitimieren. ${ }^{103}$ Bei den Anachronismen Ovids liege der Fall dagegen anders: „Those in the Metamorphoses, however, do not arouse wonder or admiration for mythology: they render it familiar. “104 Der Modus aktualisierenden Mythen-Erzählens ist folglich ein anderer als bei Vergil, weil er einem ästhetischen, primär unpolitischen Zweck untersteht, ${ }^{105}$ wodurch aber weitergehende Bezüge zu historischen oder gegenwärtigen Ereignissen nicht ausgeschlossen sind. ${ }^{106}$ Daneben weist Solodow auch auf humoristische und ironische Aspekte hin, die an so mancher Vergegenwärtigung zu Tage treten. ${ }^{107}$

Nicht ersichtlich ist aber der Grund für die Einteilung der Beispiele in Anachronismen, Romanisierungen und Modernisierungen: Auf die Problematik möglicher Überschneidungen wird zwar hingewiesen und auch darauf, dass die meisten Romanisierungen gleichfalls Anachronismen seien, ${ }^{108}$ die Zuschreibung vieler Beispiele zu einem dieser Unteraspekte erweist sich aber als wenig begründet, wenn sie ebenso gut auch in der jeweils anderen Kategorie stehen könnten. ${ }^{109}$ So scheint trotz der überzeugenden Methodik doch auch eine eher subjektive oder nicht aus der Sache selbst hervorgehende Beurteilung hinter dieser Klassifikation stehen, in der mitunter auch die in früherer Zeit $\mathrm{zu}$ beobachtende philologisch-historische Lesart durchscheint. ${ }^{110}$ Das schmälert die Beobachtungen am Text sowie die generelle Deutung des

101 So Solodow 1988, 77: „Commonly, the anachronism shrieks at us from the page.“

102 S. z. B. ebd.: „Furthermore, in a given passage the sheer quantity is sometimes persuasive.“

103 So u. a. bereits Kroll. S. o. S. 95, Anm. 40.

104 Ebd., 81. Zur Problematisierung des Begriffs „Familiarisierung“ s. o. Kap. 3.3.3.1 und u. S. 263, Anm. 212.

$105 \mathrm{Zu}$ dieser Schlussfolgerung im Zuge einer präzisen Beispielanalyse gelangt auch Classen 1981, $177 \mathrm{f}$.

106 Diesen Aspekt macht Wheeler 1999a, 197, gegen diese vornehmlich unpolitische Lesart von Solodow stark.

107 Vgl. u.a. Solodow 1988, 78 und 83.

108 Vgl. ebd., 82. Weiter unten werden dann Anachronismus und Romanisierung als „narrowest, most readily recognized forms of a general phenomenon of the poem“ (86) bezeichnet, woran sich die Beschreibung von Modernisierungen anschließt.

109 Warum z.B. ist das typisch-römische Atrium, sofern dies als solches von Ovid gemeint ist, in der mythischen Umgebung ein Anachronismus (vgl. ebd., 78), der Hinweis auf den census hingegen eine Romanisierung (83) und die Darstellung Dianas als römische Matrone eine Modernisierung (87)?

110 Um nur ein Beispiel zu geben, s. ebd., 89, über die Pythagoras-Figur im 15. Buch - besprochen im Kapitel zur Modernisierung: „The philosopher commits a stupendous error, for in his day, the late sixth century, Thebes and Athens, far from being over the hill, were nearing the acme of their greatness.“ 
Phänomens als rendering the myth familiar keineswegs, ist aber einer objektiven und ganzheitlichen Erfassung und Beschreibung letzten Endes doch in gewisser Weise hinderlich. ${ }^{111}$

Sieht man von einigen in der Folgezeit von Solodows Monographie publizierten Deutungen zu einzelnen Passagen oder Problemen ab, ${ }^{112}$ sind Tronchets „La Métamorphose à l'Euvre“ (1998) und Wheelers „Discourse of Wonders“ (1999) die nächsten ausführlicheren Beiträge zum hier verhandelten Thema. ${ }^{113}$

In Auseinandersetzung mit der an Lafaye und Grimal exemplifizierten historistischen Vorstellung weist Tronchet unter Bezugnahme auf Solodows Methodendiskussion darauf hin, dass die Figuren und Schauplätze des antiken Mythos keine historisch festgelegten Größen sind und aus diesem Grund in der von ihm als diachron bezeichneten Perspektive des heterodiegetischen Erzählers mit Attributen versehen werden können, die eindeutig für eine spätere Zeit kennzeichnend sind. ${ }^{114}$ Weil aber hierzu nur die vermeintlich in einer weit entfernten Sphäre liegenden Entitäten gezählt werden können - z. B. die architektonisch-lebensweltlichen Ausstattungsmerkmale des Göttersitzes -, ${ }^{115}$ fällt die Argumentation Tronchets bei vermeintlich Historischem zwangsläufig in eine historistische Lesart zurück. Sogenannte „certaines inadvertances ponctuelles de l'écrivain“116 seien demgemäß nicht zu entschuldigen, aber ohnehin nicht häufig. ${ }^{117}$ So heißt - dies alles steht auf derselben Seite - eine Aktualisierung im einen Fall „absichtlicher Anachronismus“, im anderen „ein Spiel aus vorgetäuschten und intendierten Inkonsistenzen“ und wiederum an anderer Stelle

Darauf, dass die Handlung - abgesehen davon, dass Pythagoras eine fiktive Figur ist - nicht „in his day“, sondern sogar zu Numas, also noch früheren Zeiten spielt und der ganze Abschnitt wohl auf nahezu jeder Seite eine historische Deutung ad absurdum führt, könnte mit gutem Recht noch aufmerksam gemacht werden. S. o. schon das Urteil von Kraus S. 100, Anm. 66 und s.u. Kap. 6.4.

111 Das ist aber auch gar nicht dessen eigentliches Ziel. Vgl. Solodow 1988, 84.

112 Hierbei zu nennen sind: Coleman 1990; Feeney 1991; Keith 1992; Schmitzer 1992; Tissol 1997; Janka 1999. Für das Buch von Granobs 1997 sei verwiesen auf die Rezension u.a. von Wheeler 1999b.

113 Wenngleich Wheelers Monographie weitaus mehr Aufmerksamkeit zuteilwurde - Tronchets gut 600 Seiten umfassende narratologische Textanalyse scheint genau zwischen Myers' und Schmitzers Forschungsüberblick auf der Strecke geblieben zu sein und wird auch nicht durchgehend in allen Bänden des neuen Ovid-Kommentars berücksichtigt - sind beide Beiträge von gleich hohem Erkenntniswert für das zu untersuchende Problem, als sie sich beide explizit auf Solodow beziehen, aber verschiedenen Hauptthesen nachgehen.

114 Vgl. Tronchet 1998, 361-364. Demgemäß handle es sich nicht um „anachronismes volontaires“ (so das an dieser Stelle kritisierte Urteil von Frécaut 1972, 64), wenn der Erzähler auf der Bildebene von Gleichnissen den eigenen zeitlich-kulturellen Standpunkt zum Ausdruck bringt.

115 Vgl. Tronchet 1998, 364.

116 Ebd., 365. Ein Widerspruch in sich ist es schon, dass dann das eigentlich als Unaufmerksamkeit kategorisierte Phänomen durch das unmittelbar genannte Beispiel (met. XV, 293-295) als absichtsvoll ausgewiesen wird, wenn in der zugehörigen Fußnote (Anm. 238) dessen Sinn für das Gesamtgedicht diskutiert wird.

$117 \mathrm{Zu}$ diesem Quantitätsargument s. wiederum das vorherige Kapitel. 
eine „unangemessene Vorwegnahme“ und „Unbeholfenheit“118, ohne dies weiter zu erläutern oder zu systematisieren.

Wenn Wheeler am Ende seiner Monographie die Annäherung des Erzählers an sein Publikum auch im stofflichen Bereich untersucht, scheinen zwangsläufig auch Strategien beachtenswert, mit denen dem Gedicht „contemporary force“ verliehen wird. ${ }^{119}$ Hierzu zählen unter anderem „color Romanus or Greek myth in Roman dress; anachronisms; and specialized concepts and diction from Roman military, civic, or legal discourse“120. Wenig nachvollziehbar ist auch hier, worin der genaue Unterschied der genannten Erscheinungen liegt bzw. warum dies anhand verschiedener Bezeichnungen spezifiziert werden muss. ${ }^{121}$ So sehr dann dieses Spannungsverhältnis aus Erzähler-Gegenwart und Gegenwart der Diegese an zahlreichen Beispielen beleuchtet wird, so wenig wird deswegen das Spezifische der Aktualisierung ersichtlich, gerade weil Aitiologien, Zukunftsprojektionen und Gleichnisse ungeachtet epistemischer Hierarchien einen ähnlichen Effekt zu erzeugen scheinen. Des Weiteren ist die These wenig überzeugend und leicht zu widerlegen, dass Ovid nur in den ersten und letzten beiden Büchern explizit Römisches in der fiktiven Welt darstelle und damit einen sozusagen zeitgenössischen Rahmen etabliere, von dem aus das Publikum auf „nuanced touches of Romanization“ im dazwischenliegenden Teil reagieren könne. ${ }^{122}$ Auch der Deutungsansatz, dass Ovid seinem Leser einen Vorgeschmack davon gebe, was im Verlauf der Erzählung noch kommen wird, geht nur in einem sehr allgemeinen Verständnis von römischer Gegenwart auf: Denn nahezu alle in den ersten Büchern genannten realen Sachverhalte haben eben keine direkte Entsprechung am Ende des

118 Alle ebd. Das letzte Beispiel, die „anticipation indue“ und „maladresse“ (gemeint ist met. XIV, 324 f.: Circes Dienstmädchen rechnet in Olympiaden), wird zudem explizit der Einschätzung Solodows, dass es sich um eine von Ovid beabsichtigte Aussage handele, entgegengesetzt.

119 Vgl. Wheeler 1999a, 197.

120 Ebd. Im selben Abschnitt ist zudem noch von „modernizing touches“ und im nächsten sowie auf den folgenden Seiten u. a. auch von „Romanizing“ bzw. „Romanization“ die Rede.

121 Auch dass an früherer Stelle schon wiederholt von „anachronisms“ (vgl. ebd., 125-139) gesprochen wird, obwohl damit in diesem Fall die tatsächlichen Anachronismen gemeint sind, die auch für Wheeler nicht zu den Strategien, eine zeitgenössische Färbung zu geben, und den auf Seite 197 mit aufgeführten, sehr wahrscheinlich als Aktualisierungen verstandenen „anachronisms“ zählen, verstärkt den Eindruck einer gewissen begrifflichen Beliebigkeit.

122 So ebd., 198f., 203 und 205 (hier das direkte Zitat). Zumal weder deutlich wird, was unter „explicitly“ genau verstanden wird, noch wie sich „heavy degree of Romanization“ (ebd., 199) gegenüber späteren, als subtiler bezeichnete Formen bestimmen lässt, noch was jene römische Gegenwart ausmacht, die in den Büchern III-XIII nicht zum Vorschein kommen soll (man denke allein an das sehr nachdrücklich, man könnte sagen heavily „aktualisierte“ achte Buch). Wieso etwa sind, um nur einen Widerspruch zu nennen, die kapitolinischen Gänse (met. II, 538f.) „the last explicitly Roman allusion“ (ebd., 198), obwohl in einer späteren Stelle noch desselben Buches die Eingeweideschau (met. II, 716f.) in einem Gleichnis sehr anschaulich vor Augen geführt wird? Richtig dagegen Farrell 2013, 249, der das concilium deorum im ersten Buch als „implicitly Roman“ bezeichnet. 
Gedichts $-{ }^{123}$ ganz zu schweigen von der Frage, ob die letzten Bücher tatsächlich von einer zeitgenössischen römischen Realität handeln und zudem noch auf eine solche Weise, dass diese an früherer Stelle antizipiert werden müsste. ${ }^{124}$ Gerade der Umstand aber, dass Aktualisierungen wie auch die anderen von Wheeler erwähnten Verfahren unterschiedslos und zum Teil mit identischen Motiven über das ganze Gedicht hinweg eingesetzt sind, lässt es unter Berücksichtigung der wenig nachvollziehbaren Gewichtung nach Explizitheit oder Häufigkeit und der geringen Differenzierung bei den einzelnen Verfahren zweifelhaft erscheinen, dass just den ersten Büchern dahingehend eine gesonderte Funktion gegenüber den späteren zuzuschreiben sei. ${ }^{125}$ Denn die Frage, ob wirklich alle Formen der Aktualisierung zugleich Romanisierungen sind und in politisch-sozialer Weise zeitgenössische römische Realität repräsentieren, bleibt dabei unberührt, wie auch eine exakte Beschreibung der einzelnen Textstellen nicht immer angestrebt wird. ${ }^{126}$

Im Allgemeinen deckt sich die Interpretation der meist als Romanisierung oder als „Roman touch“ bezeichneten Aktualisierungen mit der von früheren Arbeiten, wonach diese der Vergegenwärtigung der mythischen Welt und dem Wachhalten von derjenigen Gegenwart dienten, von der aus der vornehmlich griechische Sagenstoff betrachtet wird. ${ }^{127}$ Der entscheidende Zusatz von Wheeler ist aber der, dass dies schon in der performativen, unmittelbar ein Publikum involvierenden Anlage des Textes begründet zu sein scheint, womit die allenthalben als Veranschaulichung erklärte

123 Die Götterversammlung im ersten Buch z.B., die wie eine Senatssitzung erscheint, ist wohl schwerlich - um diese Argumentation einmal konsequent zu Ende zu denken - ein „preview“ des Senates, in dem, wie es explizit im letzten Buch heißt, Caesars Ermordung stattfindet. Feeney 1999, 27, weist dagegen noch auf Ocyrhoes Äskulap-Prophetie in met. II, 642-654, die tatsächlich als ein vereinzelter Vorverweis in diesem Sinn aufgefasst werden kann. Diese intratextuelle Verknüpfung würde aber nicht unter „Roman allusion“ nach Wheelers Auffassung fallen.

124 Die Diktion erinnert sehr an die bereits zitierte Aussage Lafayes von Ovids Ungeduld, nicht auf das 14. Buch warten zu können. S. o. S. 96, Anm. 45. Die offensichtlichen inhaltlich-strukturellen sowie thematischen Parallelen zwischen den Anfangs- und Endbüchern der „Metamorphosen“ sind nicht zu übersehen. Damit wird aber keineswegs eine irgendwie zeitgenössisch zu nennende Realität in den Schlussbüchern vorweggenommen. Sie existiert dort schlicht nicht in einer solchen Konkretheit, wie sie diese Argumentation nahelegt - allenfalls höchst implizit in der Anrede an Augustus, in der aber eine andere zeitliche und thematische Perspektive zum Tragen kommt oder selbst wiederum nur basierend auf Aktualisierungen (v. a. bei den sororum tecta trium (met. XV, 808f.); s.u. Kap. 7.3.2.1b, א). Präziser ist dies bei Feeney 1999, 27, der dies als proleptische Verweise auf die reale augusteische Zeit ohne den innertextlichen Bezug auffasst. Problematisch auch Jansen 2009, 265, die die Bücher 14-15 der „Metamorphosen“ tatsächlich als „Ovid’s present“ bezeichnet, obwohl gerade die Zeit im 14. und in weiten Teilen des 15. Buches auch dem Römer wohl eine Gegenwart war, wie wohl für den heutigen Betrachter der unterm Kyffhäuser schlafende Barbarossa gegenwärtig ist. Vgl. dazu auch Schmitzer 2006, $36 \mathrm{f}$. (Anm. 13).

125 Es wird ja sogar betont, dass es kaum eine Passage in den „Metamorphosen“ gebe, die nicht „Roman touches“ (Wheeler 1999a, 203) erkennen lässt. S. dazu auch das bereits zitierte Urteil von Otis, dass Ovid alle Mythen in ziemlich gleichem Stil und gleicher Form darbietet. S. o. S. 92, Anm. 21. 126 S. zum Thema „Romanisierung“ auch u. Kap. 7.2.2.1c.

127 Vgl. Wheeler 1999a, 205. 
Aktualisierung noch stärker in ihrer Bedeutung für den Rezeptionsvorgang selbst ins Spiel gebracht werden kann. ${ }^{128}$

In ihrer Auseinandersetzung mit Anachronismen konnten Tronchet und Wheeler neben anderen zeitgleich erschienenen Aufsätzen ${ }^{129}$ zweifelsohne Licht auf eine lange Zeit beredt übergangene Besonderheit der „Metamorphosen“ werfen. ${ }^{130}$ Die Diskussion des Problems nimmt bei Wheeler ihren Ausgang bei der Feststellung eines Gegensatzes, der zum einen in der logischen Kontinuität des Gedichts liegt, wie sie sich im Proöm durch das In-Aussicht-Stellen eines carmen perpetuum (met. I, 4) und durch die hierbei implizierte metapoetische Einbettung in einen universalhistorischen Diskurs manifestiert, und zum anderen in den Strategien des Erzählers, mit denen die Linearität eines in sich stimmigen Zeitrahmens problematisiert und infrage gestellt wird. ${ }^{131}$ Durch dieses Spannungsverhältnis werde der Rezipient beständig dazu angehalten, den faktischen Aussagegehalt als unsicher wahrzunehmen sowie das eigene Verständnis von Zeit und das der darin dargebotenen Geschichten zu hinterfragen. ${ }^{132}$ Der Erzähler hingegen lasse anhand beabsichtigter Inkonsistenzen nicht nur seine Kenntnis unterschiedlicher mythologischer Überlieferungen durchblicken, sondern weise damit zugleich auf seine eigene Unzuverlässigkeit hin, womit sich die dadurch entworfene Welt als künstlich geschaffen und deswegen selbst als wandelbar erweise. ${ }^{133}$ Tronchet hingegen geht aus von der eher traditionellen Feststellung, dass die

128 S. dazu auch u. Kap. 7.2.2.1.

129 Feeney 1999; Zissos/Gildenhard 1999 und in der Folge mit jeweils verschiedenem Schwerpunkt Hardie 2002c; Tissol 2002; Wheeler 2002; Zissos/Gildenhard 2004; O’Hara 2007, der sich vornehmlich auf Wheeler bezieht; Vial 2010, bes. 378-393, und zuletzt Farrell 2013. Für weitere, z. T. ältere Stellungnahmen zum Anachronismus in den „Metamorphosen“, sofern diese nicht ohnehin bereits erwähnt wurden oder noch werden, s. die Verweise bei Zissos/Gildenhard 1999, 31 (Anm. 1) und O’Hara 2007, 121 (Anm. 50 f.).

130 Dies liegt vor allem daran, dass dieses Problem in beiden Fällen aus dem Text selbst und seiner inneren Anlage zu erschließen versucht wird und nicht wie sonst üblich an der Erfüllung eines für normativ gehaltenen historischen Horizonts bemessen ist, der nicht nur wegen der mittelbaren Stellung der Diegese sekundär, von außen herangetragen ist, sondern auch keineswegs bei jedem Urteilenden konstant und in der gleichen Strenge angewandt sein muss.

131 Vgl. Wheeler 1999a, 117-125 und (bes. zu den Aspekten der Universalhistorie der „Metamorphosen“) Wheeler 2002. Zu betonen ist, dass diese Gegenstrategien nur vor dem Hintergrund einer bestehenden Kontinuität effektiv sein können, wie Wheeler es gegenüber der von ihm wohl zu eng aufgefassten und deswegen missdeuteten Ansicht Schmidts (zitiert wird: Schmidt 1991, 21) selbst hervorhebt, dass eine chronologische Zeitordnung gar keine Rolle in den „Metamorphosen“ spiele.Vgl. Wheeler 1999a, 130. Gegenteilig defizitär ist es aber auch, bei Wheeler nur den affirmativ-chronologischen Aspekt hervorzuheben (vgl. Cole 2004, 422 (Anm. 159) oder Oberrauch 2005, 114 (Anm. 24)). Der Dualismus aus Ordnung und Unordnung, welche sich naturgemäß nur vor einer Ordnung als solche erweisen kann, wird für das zweite Buch auch betont bei Zissos/Gildenhard 1999. Vgl. grundsätzlich dazu Adamik 1999; Boyd 2006 und Vial 2010, 389.

132 Vgl. Wheeler 1999a, 126. Feeney 1999, 27, nennt es eine „strategic uncertainty in his configuration of time“.

133 Vgl. Wheeler 1999a, 125f. und 128. Sehr häufig wird diese Künstlichkeit, die bei Wheeler noch hauptsächlich auf den Status der Erzählung bezogen ist, dann in der Folge als ein „rendering the poem 
Quellen- und Überlieferungssituation des Mythos uneinheitlich und teilweise widersprüchlich ist, und fügt dem sogleich hinzu, dass Ovid nicht bestrebt sei, vorhandene Widersprüche $\mathrm{zu}$ harmonisieren. ${ }^{134}$

Indem die „Metamorphosen“ unter diesen Gesichtspunkten betrachtet und die Intentionalität von zeitlichen Divergenzen vorausgesetzt werden, ist es dann sogar möglich, den Aufwand Ovids zu beschreiben, diese Irregularitäten hervorzuheben bzw. nicht zu vertuschen: Beispielsweise wenn er Kallisto, die sich noch in ein Sternbild verwandeln soll, das es offenbar schon vor ihrer Metamorphose gibt, im zweiten Buch explizit als die Tochter Lykaons ausweist. Dieses Detail steht im Widerspruch dazu, dass die Frevelhaftigkeit des Letzteren ein Buch zuvor die große Flut veranlasst, deren einzige Überlebende Deukalion und Pyrrha sind. ${ }^{135}$ Dieses nach der Logik der Erzählung unmögliche Vater-Tochter-Verhältnis erlangt dann aber, anstatt dass es als Nebenaspekt der mythologischen Überlieferung kaschiert wird, immer wieder Relevanz, z. B. dadurch, dass Kallisto in eine Bärin verwandelt wird, die Angst vor Wölfen hat, obwohl doch ihr Vater einer von ihnen ist (quamvis pater esset in illis met. II, 495). ${ }^{136}$ Eine „Flüchtigkeit“, wie sie noch Kraus pauschal für alle derartigen Fälle erkennen wollte, ${ }^{137}$ scheint in dieser mehrmaligen und pointierten, ja geradezu das Absurde unterstreichenden Verknüpfung kaum vorzuliegen. Eher lässt es auf eine „volonté démystificatrice“138 schließen, mit der neben anderen Auffälligkeiten, wie Sara Mack feststellt, die Präsenz des Erzählers in der Gegenwart des Erzählten sichtbar gemacht und wachgehalten, aber allen voran dessen pointierter, geistvoller Witz zum Ausdruck gebracht wird. ${ }^{139}$

Dass weiterhin diese Widersprüche nur in einer allzu schematischen historisierenden Interpretationshaltung problematisch sind, so Schmidts und in dessen Nachfolge Loehrs Deutung, ist zwar wie der grundlegende Ansatz, aus dem heraus sie erfolgt, durchaus richtig. Die konkreten Verwandlungen haben als Aitiologien eines extradiegetischen Naturphänomens für die diegetische Welt und die vom Erzähler darin repräsentierte fiktive Realität oft keine weitere Bedeutung. ${ }^{140}$ Darin aber allge-

uncertain on key ideological issues“ (so Harrison 2009, 409, in der Besprechung von O’Hara 2007) verstanden, womit hauptsächlich, aber nicht ausschließlich die Positionierung gegenüber einer offiziellen augusteischen Staatsräson gemeint ist. Vgl. auch Schmitzer 2016c, $435 \mathrm{f}$.

134 Hierzu wird in Rekurs auf Lafaye wiederum das Quantitätsargument erwähnt. Vgl. Tronchet 1998, 371.

135 Vgl. Wheeler 1999a, 129 und Tronchet 1998, 379.

136 Vgl. Wheeler 1999a, 129f. und Tronchet 1998, 380 f. Weiteres zur Stelle s.u. Kap. 5.1.2.3.

137 S. o. S. 100 , Anm. 67.

138 Ebd., 373.

139 Vgl. Mack 1988, 115.

140 Vgl. Schmidt 1991, 20 - 36 und Loehr 1996, 165 f. S.u. Kap. 7.2.1.1. Die Erklärung der Widersprüche aber mit „Verarbeitung verschiedener Quellen und poetisches Spiel“ (Schmidt 1991, 21) scheint einerseits der von Lafaye bemühten Vielfalt an $\mathrm{zu}$ bewältigendem Material verpflichtet (s. o. S. 97, Anm. 52), aber andererseits doch auch die Souveränität des Dichters ins Spiel zu bringen, wenn auch 
mein eine leicht missverständliche Bedenkenlosigkeit Ovids gegenüber der Chronologie zu erkennen ${ }^{141}$ oder das aitiologische Narrativ grundsätzlich von dieser loszusagen, weil sie nur metaphorisch oder sinnbildlich in Bezug zur erzählten Welt stehe, ${ }^{142}$ ist als Erklärung für dieses komplexe Gefüge ebenso wenig zutreffend: Nicht nur ist das Dargestellte mehrfach - durch die Erzählung, das Erzählen, die diegetische Realität und durch deren Verhältnis zur historischen Wirklichkeit - zu zeitlichen Ordnungsprinzipien in Beziehung gesetzt bzw. notwendigerweise darauf beruhend, was im Proöm programmatisch verankert und durch zahlreiche Markierungen, mitunter direkt auf einzelne Metamorphosen bezogen oder diese berücksichtigend, im Verlauf des Gedichts immer wieder und insbesondere in ihrer Widersprüchlichkeit evoziert wird; ${ }^{143}$ sondern auch der Effekt, der in dieser kontinuierlich erfahrbaren Frustration chronologischer und historischer Prinzipien liegt, erfordert dieses Ordnungsprinzip als Negativfolie. ${ }^{144}$

In der Schlussfolgerung, dass die „Metamorphosen“ aufgrund der beobachteten Spezifika nicht historisch gelesen werden können, stimmen die bis hierhin vorgestellten Ansätze, besonders die von Schmidt, Tronchet und Wheeler überein, mag die jeweilige Interpretation bei den Einzelphänomenen auch deutlich voneinander abweichen.

Eine fundamental entgegensetzte Auffassung diesbezüglich sowie bezüglich der Anachronismen überhaupt ${ }^{145}$ zeigt Coles Arbeit, die 2008 in Buchform, aber im Wesentlichen schon 2004 als Aufsatz publiziert wurde.

dies für Widersprüche selbst, die dem Wortlaut nach ein zufälliges Beiprodukt zu sein scheinen, nicht gilt.

141 Vgl. Herter 1982, 109f., auf den sich Schmidt 1991, 23, beruft, oder Lyne 1984, 25. Wenig klarer wird dies durch eine spätere Bemerkung von Schmidt 1991, 43: „Ovid ist unbekümmert um Chronologie, nicht um chronologische Widersprüche." Gemeint ist wohl eine historische, extradiegetische Chronologie, während die Widersprüche die von der Erzählung sind. Überdies impliziert Schmidt selbst, dass sich Ovid doch in gewisser Weise an eine Chronologie hält, wenn sie, so Schmidt kurz vorher, nicht „,wirklich immer eingehalten“ (ebd.) wird.

142 So Loehr 1996, 166.

143 Damit ist die Rezeptionsweise, die die von dem Text berichteten Ereignisse in ein chronologisches Verhältnis zueinander setzt, sowohl die naheliegende, weil naive Herangehensweise als auch eine, zu der die Erzählinstanzen beständig auffordern. Vgl. Wheeler 1999a, 118-122 und Cole 2008, 93-95. S. auch allein den einleitenden Absatz zum diesbezüglichen Kapitel bei Tronchet 1998, 329.

144 Von einem „unbefangenen Leser“ auszugehen, den die Widersprüche nicht störten, „weil sie für ihn, aufgrund seiner impliziten Prämissen, nicht vorhanden sind“ (Schmidt 1991, 21), ist - auch ohne verstanden zu haben, was „implizite Prämissen“ eines solchermaßen konstruierten Lesers sind wenig überzeugend. Denn dann könnte schlechterdings jede komplexere Sinnbeziehung eines Textes verworfen werden, wenn die Erkenntnisfähigkeit der Rezipienten auf ein Minimum reduziert werden würde (wie z. B. im „Literaturgespräch“ bei Peter Squentz und seinen Genossen; s. o. Kap. 1.1). Das gegenteilige Extrem dazu ist Cole 2004, 389, der es einem „less informed reader“ zutraut, Kephalos als Verbindungsstück der pandionidischen Genealogie zwischen Aegina und Theben auszumachen.

145 Aktualisierungen bzw. sog. kulturelle Anachronismen werden hiervon als bedeutsam unterschieden oder zumindest nicht schlechthin ausgeschlossen. Vgl. ebd., 414 (Anm. 142). Obschon dieses Urteil in der später erschienenen Monographie mit Blick auf die kulturelle Entwicklung, die in den 
Im Rekurs auf quellenkritische, fast ausschließlich aus dem späten 19. und frühen 20. Jahrhundert stammende Arbeiten, ${ }^{146}$ in denen der Frage nach der chronologischmythographischen Vorlage für die „Metamorphosen“ nachgegangen wird, weist Cole auf die zahlreichen Parallelen hin, die zwischen der Abfolge der Einzelepisoden bei Ovid und den Königslisten von Kastor von Rhodos und Varros „De gente populi Romani“ erkennbar sind bzw. rekonstruiert werden können. ${ }^{147}$ Neben den vielen Detailbeobachtungen, die in ihrer sehr anspruchsvollen Darbietung in Teilen hilfreich sind, um den zeitlichen und logischen Aufbau des Gesamtgedichts zu erschließen, offenbart jedoch nicht zuletzt die Diskussion bei den vermeintlichen Abweichungen, dass der generelle Blickwinkel in beiden Abhandlungen ein strikt historistischer ist, weil eine in sich abgeschlossene heroische Zeit, „fabularis historia“148, als Norm für die Mythendarstellung postuliert wird. ${ }^{149}$ Folglich steht die Bewertung möglicher zeitlicher Inkonsistenzen a priori im Lichte der Nicht-Erfüllung eines positivistisch betrachteten Ideals und wird eben nicht dahingehend vorgenommen, dass ein Erzähler dies in gebundener Sprache in einem fiktionalen Narrativ berichtet, dessen Wahrheitsgehalt zudem ostentativ und wiederholt bezweifelt wird. ${ }^{150}$

\footnotetext{
„Metamorphosen“ zu erkennen behauptet wird, deutlich relativiert ist: Aktualisierungen seien demnach „presumably intentional“, wenn Ovids von Cole behauptete Chronologie der Kulturentwicklung nicht verletzt werde. Aber andere, die Chronologie verletzende Beispiele seien dann entweder ,inadvertent or introduced for humorous effect“, wobei er wiederum bei den Aktualisierungen in der Zeichnung der Götter einschränkend feststellt: „These are not really violations“. Vgl. Cole 2008, 95 (Anm. 197).Wie teuer erkauft die Aufrechterhaltung einer solch evolutionär-chronologischen Lesart der „Metamorphosen“ ist, die in weiten Teilen jener Sichtweise aus den vorangegangenen Jahrhunderten gleicht, dürfte schon hier die Variabilität der herangezogenen Erklärungen, deren Beliebigkeit und deren Pauschalität deutlich vor Augen führen.
}

146 Vgl. Cole 2004, 356f. (Anm. 4-7). Vgl. den zusammenfassenden Kommentar zu diesen früheren Arbeiten bei Bömer 1969, 7 und die Erläuterungen bei Bömer 1977, $275 \mathrm{f}$.

147 Eine detailliertere und ausgewogenere Diskussion der Quellenfrage bei den „Metamorphosen“ liefert Cameron 2004, 261-303.

148 Vgl. u.a. Cole 2008, 14.

149 Dass dabei der dichterische Formwille nur peripher von Bedeutung ist, scheint auch Cole implizit in Kauf zu nehmen, wenn er den Hinweis von Kraus zitiert, dass man Ovid nun (= im Jahr 1968!) aufgrund der ihm zuerkannten Selbstständigkeit weniger in Abhängigkeit von seinen Quellen sehen dürfe, aber letztlich dennoch an jene frühere, Ovids Abhängigkeit betonende Tradition anknüpft. Vgl. Cole 2004, 356 (Anm. 5). Überdies unterstreicht die bereits früher zitierte Bezugnahme Coles auf Lafaye diese Positionierung. S. o. S. 97, Anm. 53. Vgl. zudem ebd., 363, wo das Abweichen Ovids von Kastor allein mit dem Einfluss einer anderen Quelle zusammenzuhängen scheint.

150 Sehr stark an Eberts (s. o. S. 92, Anm. 20) oder Krolls (s. o. S. 95, Anm. 40) Argumentationsweise erinnert die Erläuterung, dass das Wissen, das die Minyas-Töchter für ihre Geschichten heranziehen, daher stamme, dass sie vermutlich phönizische Freunde oder Verwandte gehabt hätten. Vgl. ebd., 370 (Anm. 44). Eine Antwort darauf, wer dann der ersten Minyade von römischen Wasserleitungen berichtet haben könnte - in den Augen mancher sogar „il più clamoroso anacronismo del poema“ (Barchiesi/Rosati 2007, ad met. IV, 122-124) -, bleibt ein solches Erklärungsmuster aber notwendigerweise schuldig. Für „neoterische“ Kosmetikgewohnheiten bei der dritten Minyas-Tochter s. außerdem u. Kap. 5.2.2. 
Dass dies beim Anachronismus zudem weder kategorisch, d. h. basierend auf einer Formulierung von sachlichen Kriterien, durch die sich dieses Phänomen gegenüber anderen auszeichnet, noch systematisch in Bezug auf den Gesamttext geschieht, vermittelt eine eher subjektive, sehr selektive Sicht auf die Problematik: So wird es als „only significant bit of chronological dislocation“ angesehen, dass Kirkes Dienerin in Olympiaden rechnet - auch wenn, so der erläuternde Zusatz, von einem „member of the Italian servant class“ ohnehin nicht mehr erwartet werden könne. ${ }^{151}$ Wodurch ausgerechnet diese Auffälligkeit, die zudem zwar von dieser Dienerin „begangen“, aber eigentlich in Form eines hypodiegetischen Berichts vermittelt durch den homodiegetisch-intradiegetischen Erzähler Makareus erzählt wird, ${ }^{152}$ einzigartig ist, erschließt sich nicht. ${ }^{153}$ Warum sie sich ferner von den anderen unterscheidet, die von Cole im Kontext der Pythagoras-Episode durchaus auch erwähnt werden; ${ }^{154}$ und wieso just diese eine bedeutsam ist, ${ }^{155}$ aber alle anderen, soweit erkannt, als unabsichtlich, als in ihrer katalogmäßigen und ekphrastischen Darbietung entschuldbaren ${ }^{156}$ oder als unspezifische oder mit einer vielsagenden Zurückhaltung doch als irgendwie signifikante Erscheinungen diskutiert werden, bleibt trotz oder gerade wegen des apodiktischen Tenors gänzlich unklar. ${ }^{157}$

151 Cole 2004, 410. Was hier noch als Fehler bezeichnet ist, dem aber letztlich doch Sinn abgewonnen werden kann, wird dann in der Monographie bei den „,beginnings of an accurate system for measuring time“ herangezogen und nur in der Fußnote unter z. T. wortwörtlicher Wiederholung des früheren Artikels als „in character of the speaker“ weitaus neutraler erklärt. Vgl. Cole 2008, 94 und $94 \mathrm{f}$. (Anm. 196).

152 Wodurch diese historistische Erklärung, eine italische Dienerin hätte es eh nicht besser wissen können, substanzlos ist. Denn wer, wenn nicht ein Grieche - so ließe sich der Gedanke fortsetzen, will man sich auf eine solche Argumentation überhaupt einlassen - hätte dies sofort als falsch erkennen müssen und niemals kommentarlos wiederholen dürfen (abgesehen davon, dass natürlich auch dieser Grieche es ohne prophetische Gaben noch nicht hätte wissen können)? Vgl. zudem Myers 2009, ad met. $\mathrm{XV}, 324-325$ : „the nymph is presumably Greek, as is her auditor Macareus“.

153 Ungeachtet dessen, ob dies als Anachronismus anzusehen ist. S.u. Kap. 5.2.

154 Natürlich gilt dort: „Ovid himself must bear the blame“ (Cole 2004, 410).

155 Was ist dann mit Herakles in Buch 11, laut Ludwig - obwohl auch ein solches Urteil letzten Endes kaum objektivierbar ist - „die stärkste chronologische Diskrepanz im Aufbau der Metamorphosen“ (Ludwig 1965, 60)?

156 Was sich nebenbei bemerkt damit widerspricht, dass Cole andernorts dieser Darstellungsweise sehr viel Bedeutung beizumessen weiß, wenn diese mit der eigenen Argumentation übereinstimmt. Vgl. z. B. Cole 2004, 387 und Cole 2008, 22, wo die Aktualisierung bei met. VI, 414 nicht erkannt und das Epitheton positivistisch als Textbeleg verwendet wird (auch Rosati ${ }^{2} 2013$, ad met. VI, 412-423, folgt dieser Lesart und nimmt keinen Anachronismus bzw. keine Aktualisierung wahr). S.u. Kap. 6.2.

157 Erwähnenswert ist hierbei auch die Art des Umgangs mit manchen der weiter oben genannten, dem historistischen Standpunkt völlig gegensätzlichen Forschungsbeiträgen. So werden einige Titel zwar zitiert, aber meist so, als ob deren Kernthese im Einklang mit der eigenen Argumentation stünde (u.a. Zissos/Gildenhard 1999 in Cole 2004, 374 (Anm. 55)). Wie verzerrend dies ist, zeigt sich sehr gut bei ebd., 370 (Anm. 45), wo zwar richtigerweise mit Feeney 1999, 15, die Ansicht bestärkt wird, dass Ovid Varro verpflichtet sei. Entscheidend ist jedoch der Kontext, in dem dies Feeney selbst behauptet; denn bei ihm heißt es wenige Seiten später [Hervorh. v. Verf.]: „Now, having sketched the chronographic 
Die durch den Aufsatz vorbereitete und dann in der Monographie präsentierte These, Ovid stelle eine Mythenhistorie dar, die sich in einem evolutionären Prozess von Buch zu Buch bis zur Zeit des Augustus als Zustand der Vervollkommnung entwickle, ${ }^{158}$ und dem Text sei aus diesem Grund eine historisch-didaktische Zielsetzung zu eigen, ${ }^{159}$ ist daher nicht überzeugend. Diese Auffassung scheint zudem nur möglich, weil störende Elemente darin entweder sehr unsystematisch und uneinheitlich subsumiert, ${ }^{160}$ schon im Vorfeld ausgeklammert oder gar nicht berücksichtigt werden. ${ }^{161}$

Der Ansatz liefert folglich aufgrund der methodischen Probleme, die im Wesentlichen dieselben wie die der bis einschließlich aus dem frühen 20. Jahrhundert stammenden Arbeiten sind, für den Anachronismus kaum neue Einsichten, eher ist eine systematische Erfassung wegen der Lücken und Widersprüche in der Argumentation sogar erschwert. ${ }^{162}$

models at Ovid's disposal, I need to say that Ovid ignores, refutes, renounces all such schemes and ideologies, or else subverts the canonical reference-points that no account of history could totally ignore." (ebd., 18). Ähnlich irreführend ist dies, wenn Cole bei Tronchet ausschließlich betont, wie sehr dieser die Bedeutung der chronologischen Untermauerung des Gedichts und deren Stellung für dessen Einheit herausstellt (vgl. Cole 2004, 366 (Anm. 41)), ohne Tronchets differenziertere Sicht auf chronologische Irregularitäten und Anachronismen zu erwähnen, in der er diesen einen berechtigten Platz in einer global zu verstehenden Einheit sehr entschieden einräumt (vgl. allein die Zusammenfassung in der Einleitung bei Tronchet 1998, 26) - von dessen Kritik an einer historistischen Deutung der „Metamorphosen“ gar nicht erst zu sprechen (vgl. ebd., 361).

An späterer Stelle werden auch Gegenpositionen wiedergegeben - die von Schmidt, Lyne und Solodow, von denen die ersten beiden nur in der verkürzten Sichtweise als das ungenaue Extrem zu Coles Auffassung erscheinen, während Solodow, wie oben bereits erläutert wurde, nur eine überaus knappe Bemerkung dazu macht. Vgl. Cole 2004, 416 (Anm. 148). Die neuere, mit Tronchet und Wheeler einsetzende Lesart bleibt - anders als es die Zitationsweise erscheinen lassen möchte - allerdings nahezu unerwähnt oder wird sehr pauschal als falsch abgeurteilt. Vgl. Cole 2008, 9 f.

158 Den zuweilen verwendeten Begriff „collective Bildungsroman“ (ebd., 78) allein von der Gattung her zu kritisieren, übersteigt den hier gebotenen Rahmen. Als uneigentliches Sprechen - auch von Albrecht 2014, 165, weist darauf hin, dass dieser Begriff „nur metaphorisch“ aufgefasst werden könne ist dies ohnehin problematisch, wie in einem anderen Kontext schon bemerkt wurde. S. o. S. 57, Anm. 167.

159 Vgl. Cole 2008, 13f. Man denke dagegen nur an die Präfiguration einer „Metamorphosen“-Poetik in am. III, 12, in der die historica fides explizit verworfen wird. S. o. Kap. 3.1.2.

160 S.u. a. oben S. 78, Anm. 278; unten S. 189, Anm. 96; S. 190, Anm. 102; S. 191, Anm. 104; S. 195f., Anm. 123 und S. 350, Anm. 619.

161 S.u. a. oben S. 41, Anm. 71; unten S. 171f., Anm. 23; S. 172f., Anm. 29 und S. 173, Anm. 34. Das affirmierende Aufgreifen von Coles Lesart durch Farrell 2020 lässt diese erheblichen Widersprüche unbeachtet; gleichermaßen ist es zu einfach, im Jahr 2020 als Gegensatz zu Coles Ansatz von der durch zahlreiche substantielle Aufsätze und Monographien vertretenen communis opinio einzig den Aufsatz von Zissos/Gildenhard 1999 zu nennen (vgl. Farrell 2020, 325).

162 Dies beurteilt ausreichend schon Schmidt 1991, $20 \mathrm{f}$, dessen Aussage, kein moderner gelehrter Leser habe wohl Historizität im modernen Sinn und strikte chronologische Folge allen Ernstes behauptet, wohl aber nicht nur die frühere Forschung zu wohlwollend auffasst, sondern sich noch durch Coles Buch als zu zuversichtliches Postulat herausstellen sollte. 


\subsection{3 „Ein Anachronismus, den man nicht unnötig bemühen sollte“ - die „Metamorphosen“-Kommentare}

Philologische Kommentare, deren Ziel es ist, ein Gedicht vom Umfang der „Metamorphosen“ in seiner Gesamtheit zu erläutern, an einem einzelnen Motiv zu beurteilen, ist nicht nur wegen dieser Spezialisierung ungerecht, sondern auch, weil eine kritische Revision in ihrer Perspektive zeitlich privilegiert und übermäßig genau sein kann. Diese diachrone und gewissermaßen diatextuelle, alle Kommentarbände insgesamt berücksichtigende Betrachtungsweise ist aber gerade deshalb für ein allgemeines Bild zu Anachronismen und Aktualisierungen sehr aufschlussreich. ${ }^{163}$

In Bömers Kommentar sind zeitliche Widersprüche - das dürften die bisherigen Zitate $^{164}$ deutlich zeigen - etwas vorwiegend Negatives, idealerweise zu Vermeidendes. Eine Begründung, weswegen eine nicht-anachronistische Lesart in einem solchen Fall vorzuziehen und die gegenteilige Auffassung nicht zu „bemühen“ sei, obwohl andere ähnliche Stellen vielfach von ihm selbst als Anachronismus bezeichnet sind, wird nicht gegeben. Gleichermaßen kann aus der anderen zitierten Aussage, dem Dichter sei bei seiner vermeintlich anachronistischen Beschreibung der diegetischen Umgebung „die Phantasie durchgegangen“"165, nur implizit geschlossen werden, dass diese in den Augen des Kommentators wohl dann das Richtige trifft und angemessen ist, wenn die kulturellen und historischen Besonderheiten des Handlungsschauplatzes rekonstruierend berücksichtigt sind oder dessen Zeichnung semantisch so unvollständig ist, dass das Realitätsprinzip nicht durch eindeutig fremde und in diesem Sinn befremdliche Attribute eingeschränkt wird. ${ }^{166}$ Offensichtlich ist daran auch, dass Bömer den Mythos überwiegend historisch beurteilt: ob dies nun die sozialen Verhältnisse in Milet betrifft, deren genaue Nachzeichnung der Phantasie des Dichters zum Opfer fällt, oder sonstige Ausstattungsmerkmale, Gebräuche oder auch die Chronologie der erzählten Welt. ${ }^{167}$ Zwei Aspekte seien für Bömers generelle Einschätzung zum Anachronismus noch genauer vorgestellt: zum einen die Terminologie, mit der zeitliche Auffälligkeiten beschrieben werden, und zum anderen das, was darunter genau gefasst wird.

163 Hierfür wurden nur die maßgeblichen Gesamtkommentare berücksichtigt - s. hierzu das Urteil bei Schmitzer 2007, 157f. -, wobei diejenigen zu einzelnen Büchern dann bei den entsprechenden Textstellen konsultiert werden. Für die „Metamorphosen“-Ausgabe von Haupt/Ehwald s. o. Kap. 4.1.1. 164 Das im Titel dieses Kapitels stehende Zitat stammt aus Bömer 1969, ad met. I, 287.

165 S. o. S. 90, Anm. 1. Eine neutralere Einschätzung zum Abwandeln einer vermeintlichen historischen Realität findet sich dagegen dann bei Bömer ${ }^{2} 2008$, ad met. VI, 412.

166 S. dazu die Ausführungen in Kap. 3.2.3.

$167 \mathrm{Zu}$ den Ähnlichkeiten mit Servius s. o. Kap. 3.1.3. Was aus methodischer Sicht aus den mehrfach genannten Gründen ungenau ist, erweist sich für die Ermittlung der beachtenswerten Textstellen als Glücksfall. Denn Bömers Akribie im Zusammentragen und Dokumentieren von Anachronismen gerade die Auflistungen unter der Rubrik „Anachronismus“ in den späteren Bänden (vgl. Bömer ${ }^{2} 2016$, ad met. XI, 131f.; Bömer ${ }^{2}$ 2006, ad met. XII, 102-104 und Bömer 1986, ad met. XIV, 183f.) - ermöglicht einen sehr umfassenden und zuverlässigen Zugang zu diesem Phänomen. 
Die große Varianz an verschiedenen Ausdrücken ist allein beim Umfang des Kommentars nicht verwunderlich, und das „unbekümmerte“168 und „unbedenkliche“169 Übertragen von Zeitgenössischem in die mythische Früh- oder Vorzeit erinnert an jene traditionell vertretene Auffassung: ${ }^{170}$ Neben „Anachronismus“ oder „poetischer Anachronismus“ ist häufiger auch von einem „Zusatz ex persona poetae“ bzw. „ex ore et more Ovidiano“ sowie von einem „Widerspruch in der Chronologie“ die Rede. ${ }^{171}$ Zuweilen heißt es aber auch ganz unspezifisch, dass „Ovid anachronistisch“ an etwas „gedacht hat“172. Was aber positiv vor allem gegenüber Arbeiten jüngeren Datums auffällt, ist die Vermeidung von Ausdrücken wie Modernisierung oder jene ab Solodow so häufig verwendete Romanisierung. ${ }^{173}$ Als interessante Eigenart ist noch zu nennen, dass Bömer trotz der zum Teil sehr deutlichen Wertungen mancherorts einen gewissen Vorbehalt andeutet, wenn entweder einschränkende Zusätze ${ }^{174}$ oder Anführungszeichen ${ }^{175}$ die Assertion seiner Aussage relativieren bzw. zu relativieren scheinen oder eine letztendliche Festlegung bewusst unterbleibt: Demgemäß ist es dann ,,[p]oetische Ungenauigkeit‘ [...] oder Anachronismus“"176.

168 Bömer 1977, ad met. IX, 671. Durch die nachfolgende Klammer mit dem Verweis auf einen anderen „Anachronismus“ wird dies zudem auch als solcher ausgewiesen.

169 Bömer 1969, ad met. II, 326.

170 Auch hinsichtlich der innertextlichen Chronologie wird die Gleichgültigkeit Ovids, wie sie schon Kraus feststellt und dann gerade Schmidt wieder stark macht (s. o. Kap. 4.1.2), hervorgehoben (vgl. Bömer 1977, 276) oder in ähnlich süffisanter Weise wie bei Haupt/Ehwald (s. 0. S. 94, Anm. 35) umschrieben: „Über solche chronologischen Fragen hat der Dichter sich den Kopf nicht zerbrochen“ (Bömer 1969, ad met. II, 713). Interessanterweise zitiert aber Bömer andernorts (Bömer ${ }^{2} 2016$, ad met. XI, 131f.) kommentarlos - wenn auch leicht abgeschwächt durch Zusatz eines „gegebenenfalls“ - auch die Aussage von Ulrich von Wilamowitz-Moellendorff, der feststellt, dass „Ovid gescheit genug ist, sich nicht vor Anachronismen zu scheuen“ (von Wilamowitz-Moellendorff 1924, 242).

171 In der obigen Reihenfolge: Bömer 22008, ad met. VI, 416; Bömer 1969, ad met. II, 296; ebd., ad met. I, 200; Bömer 1986, ad met. XV, 359 und Bömer 1969, ad met. II, 713. An einer Stelle wird „Anachronismus“ auch direkt mit der antiken Bezeichnung anticipatio bei einer Servius-Stelle in Verbindung gebracht. Vgl. Bömer 22008, ad met. VI, 417. S. o. S. 59f., Anm. 179.

172 Bömer ${ }^{2} 2016$, ad met. XI, 626-629.

173 Ausgenommen ein direktes Zitat von Sauvage, bei dem eine Aktualisierung eine „romanisation du thème“ (Sauvage 1975, 49) genannt wird (wohlgemerkt nur allgemein in Bezug auf die carceres im circus, aber nicht in Bezug auf die „Metamorphosen“ und auch ohne diese als Beispiel zu zitieren). Vgl. Bömer 22016, ad met. X, 652f. Gleichfalls wird ein einziges Mal Vergils Behandlung der italischen Frühgeschichte als „Romanisierung“ (dort in Anführungszeichen) bezeichnet. Vgl. Bömer 1986, ad met. XIV, 449f.

174 So ist es „eine Art Zusatz ex persona poetae“ (Bömer 1969, ad met. I,172f.) oder ist „,sozusagen anachronistisch“ (ebd., ad met. II, 710).

175 So wird Anachronismus einmal ohne (ebd., ad met. II, 796), andernorts mit Anführungszeichen (Bömer 22011, ad met. V, 399) erwähnt; weiterhin heißt es im einen Fall „poetischer ,Anachronismus““ (Bömer 1969, ad met. II, 171), bei dem nur „Anachronismus“ in Anführungszeichen steht, im anderen Fall aber beides (ebd., ad met. II, 252).

176 Bömer ${ }^{2} 2011$, ad met. IV, 772. Zu beachten sei auch hier die (signifikant?!) unterschiedliche Verwendung der Anführungszeichen. 
Dieses Nebeneinander von überzeugter Stellungnahme, Abschwächung der Behauptung und betonter Zurückhaltung im Bereich der Terminologie findet seine Entsprechung auch auf der begrifflichen Seite: Weder erfolgt eine wirkliche Unterscheidung zwischen Anachronismen und Aktualisierungen - anders als z.B. noch Ebert, der tatsächliche Anachronismen als irrelevant ausschließt, verwendet Bömer „Anachronismus“ für beides - noch ist dabei offenbar der epistemische Status der Erzählung ausschlaggebend, da die Bildebene von Gleichnissen wie auch jene unter die Kategorie Anachronien fallenden Prolepsen ebenso zu Anachronismen gezählt oder in deren Nähe gerückt werden. ${ }^{177}$ In manchen Fällen scheint überdies das mit einem Objekt verbundene Realienwissen so dominant zu sein, dass schon eine vermutlich nur als Analogie zu definierende Ähnlichkeitsbeziehung bereits ein „Anachronismus" ist. ${ }^{178}$

Ein zusätzliches Problem ist es, dass diese Haltung innerhalb der Kommentarbände selbst uneinheitlich ist, weil Definitionen gegeben oder erläuternde Angaben gemacht werden, die dem eigenen Umgang mit dieser Klassifikation widersprechen, ${ }^{179}$ oder dass sich diese Haltung durch mehrere Einzelbemerkungen als deutlich vielschichtiger erweist, als es die Kommentierung an den jeweiligen Einzelstellen besonders in den früheren Bänden vermuten lässt. ${ }^{180}$ Denn so wertvoll die ergänzenden Informationen dann eigentlich sind, ${ }^{181}$ umso schwieriger ist es, durch sie einen schlüssigen Gesamteindruck zu gewinnen, weil verschiedene Textphänomene gleich oder gleiche verschieden bezeichnet sind; oder weil tatsächlich vorhandene Erläuterungen zu diesen Bezeichnungen eher beiläufig und allgemein sind. Setzt man diese terminologischen Ambivalenzen zu der grundlegenden interpretatorischen Haltung des Kommentators in Beziehung, zeigt sich ein weiteres Defizit: Weil die Perspektive vorwiegend historisch ist, leistet ihre Beschreibung keine neutrale kategorische Klassifizierung, anhand derer Ähnliches rubrikartig zusammengestellt wird, sondern

177 Vgl. zu Ersterem (Gleichnisse) Bömer 1977, ad met. VIII, 282f.; ad met. VIII, 357 f. (wo dies mit Zitat von Hollis als ,Brechen mit der heroisch-griechischen Atmosphäre‘ erklärt wird); ad met. IX, 217f.; Bömer 22016, ad met. XI, 508f.; Bömer 22006, ad met. XII, 102-104; Bömer 1986, ad met. XIV, $183 \mathrm{f}$.

Was die Prolepsen betrifft: Wie auch tatsächliche Aktualisierungen sind temporal markierte Erzählerverweise als ein „Zusatz ex persona poetae“ bezeichnet. Vgl. Bömer 1969, ad met. II, 259, hier ein Vorverweis in einem „Damals“ der mythischen Zeit.

178 Vgl. Bömer 22006, ad met. XII, 254f., wo es um einen Tisch aus Ahornholz geht, von welchem Baum in der römischen Antike offenbar „ein beliebtes, aber nicht das wertvollste Holz für Tische“ gewonnen wurde.

179 Z. B. ebd., ad met. XII, 102-104, wo mit dem Hinweis auf von Albrecht 1981, 2337, stichwortartig die „Schranke zum Anachronismus“ bei den Gleichnissen erwähnt wird.

180 Z. B. bei Bömer 1969, ad met. II, 176, wo - nachdem diese Bezeichnung schon zuvor mehrfach verwendet wurde - zum „Zusatz ex persona poetae“ gesagt wird: „Der Dichter spricht, auch in der Form von Anspielungen, von Dingen, die er ohne Anrede, aus seiner Sicht, dem Geschehen der Vergangenheit hinzufügt, ein Mittel, die Darstellung lebendiger zu gestalten.“ Es ist nicht nur vage, was unter einer „Anspielung“ und „seiner Sicht“ zu verstehen ist, sondern es hängt überhaupt vom Zufall ab, ob ein normaler Kommentarnutzer auf derlei Erläuterungen stößt.

181 S. hierzu auch die Bemerkung bei Schmitzer 2007, 159. 
drückt zugleich eine interpretatorische Festlegung aus. Wenn im obigen Beispiel die Frage, ob „poetische Ungenauigkeit oder Anachronismus“ vorliegt, eine Antwort offenlässt oder davor gewarnt wird, eine Erklärung für einen vermeintlich anachronistischen Textbefund nicht „unnötig“ als solche zu „,bemühen“, kann diese Zurückhaltung den Blick auf das Phänomen trüben.

Dadurch aber, dass der Bömer-Kommentar eine umfassende Sammlung an Beispielen für Anachronismen, Aktualisierungen und Synchronismen generieren konnte, dass ferner vielfach weitergehende Deutungen vorgebracht und Denkanstöße für spezialisiertere Fragestellungen zu diesem oder einem angrenzenden Thema gegeben werden und dass die z.T. kontroverse oder variierende Bewertung von einzelnen Textstellen geradezu dazu einladen, dieses Phänomen mit einer deduktiven und systematischen Methodik neu zu beleuchten, bildet er den unverzichtbaren Ausgangspunkt für diese Untersuchung.

Eine hilfreiche Ergänzung hierzu bietet sich durch die von Alessandro Barchiesi und anderen herausgegebene Kommentarreihe, die neuere Entwicklungen der OvidPhilologie berücksichtigt ${ }^{182}$ sowie über den Index im letzten Band einzelne Stellen leichter auffindbar macht.

Ähnlich wie bei Bömer dient anacronismo als Sammelbegriff für entsprechende zeitliche Auffälligkeiten. Die Überschneidungen mit anderen Lemmata wie romanizzare, wozu jegliche Referenz auf Rom oder Römisches gezählt wird, und den gleichermaßen unspezifischen paradosso oder cronologia legen aber nahe, dass Anachronismus und ähnliche Phänomene nicht anhand festgelegter Kriterien definiert sind, sondern durch ihr Vorkommen im Text jeweils als das, wofür es im konkreten Fall gehalten wird, ausgewiesen sind. ${ }^{183}$ Ein systematischer Blick auf die konkret verwendeten Bezeichnungen ist deshalb wenig ertragreich, wenngleich Unterschiede bei den einzelnen Bänden und ihren Herausgebern erkennbar sind. ${ }^{184}$

182 Vgl. Barchiesi ${ }^{4} 2013$, CXXXIIIf.

183 Das hat zur Folge, dass Textstellen, die von den Kommentatoren nicht explizit als anachronistisch oder dementsprechend bezeichnet werden, auch nicht im Register auftauchen (v. a. Argo und Troja). Markant ist dies z. B. bei Barchiesi ${ }^{4} 2013$, ad met. II, 795, wo nur eine ironische Anspielung ausgemacht wird, aber auf die Aktualisierung selbst nicht eingegangen wird, während hingegen der vorherige Vers aufgeführt wird, weil dort von „anacronistico“ die Rede ist. Auch das gleiche Motiv, das im einen Fall unter „Romanisierung“ aufgelistet ist, bleibt im anderen Fall im Register zu eben jener Romanisierung unerwähnt, weil an der entsprechenden Stelle nur von einer „assimilazione“ (ebd., ad met. II, 296) gesprochen wird. Vgl. auch Reed 2013, ad met. X, 595, der bei einem Gleichnis von einer ,interpretazione specificamente romana“ spricht.

184 Interessant mag es aber sein, dass nicht nur das, was als Anachronismus angesehen wird, keiner konkreten Definition unterliegt (wenngleich an einer Stelle eine „categoria di anacronismi“ als „confusione die piani temporali all'interno del racconto“ (Barchiesi ${ }^{4} 2013$, ad met. 538-541) genannt wird), sondern auch die Bezeichnungen, mit denen das Phänomen dann beschrieben werden soll, scheinbar beliebig variieren: neben jenem ,più clamoroso anacronismo del poema“ (Barchiesi/Rosati 2007, ad met. IV, 122-124) ist u.a. die Rede von „evidenti anacronismi“ (Rosati ${ }^{2} 2013$, ad met. VI, 70 128); dagegen findet sich aber auch „anacronisticamente“ (Reed 2013, 222) und sogar „un tocco ,anacronistico““ (Kenney 2011, ad met.VII, 239f.). Wie bei Bömer werden zuweilen Anführungszeichen 
Erschwert die fehlende Systematik ohnehin aus den bekannten Gründen ein exakteres Verständnis, so zeigt sich insbesondere bei deren Deutung als Romanisierung ein entscheidender Nachteil gegenüber der Vorgehensweise bei Bömer. Wie die allgemeinen Bemerkungen unter dem Stichwort „Romanisieren, Hellenisieren“ in der Einführung zu Beginn des ersten Bandes deutlich machen, sind zum einen der Kulturtransfer von Griechischem ins römische Geistesleben und zum anderen das für Ovid zeitgenössische augusteische Rom wichtige Interpretationsschwerpunkte für den Kommentar. ${ }^{185}$ Für den Blick auf Anachronismen und Aktualisierungen bedeutet das aber, dass die Deutung des Befundes Gefahr läuft, an die Stelle seiner Beschreibung zu treten. ${ }^{186}$

Hierzu als Beispiel nochmals die Götterversammlung im ersten Buch. Nach einer motivgeschichtlichen Einordnung zum concilium deorum werden die Rom-Analogie in der Ortsbeschreibung in met. I, 168-176 und die sprachlichen Mittel, die diesen Eindruck erzeugen, herausgearbeitet. ${ }^{187}$

Eine realistische, zeitgenössische Darstellung der Götterversammlung sei schon bei Homer klar erkennbar, allerdings verdeutliche Ovid diesen Zug, indem er ihn eindeutiger auf nur eine Stadt, nämlich Rom, münzt. ${ }^{188}$ Neben anderen Ausdrücken seien hierfür auch die atria „un altro importante segno di romanizzazione“ ${ }^{\text {"189, }}$, ohne

(als Einschränkung?) verwendet, aber nicht konsequent und ohne Angabe eines Grundes. Weiterhin findet sich das gänzlich unpräzise „anachronistisch anspielen“ (vgl. ebd., ad met. VIII, 282f. (in Bezug auf ein Gleichnis) und IX, 466 und Hardie 2015, ad met. XIII, 251f.) sowie in einem Fall sogar „paragoni anacronistici“ (Kenney 2011, ad met. VIII, 357 f. (mit Verweis auf Hollis 1970, ad loc.)), obwohl das an der Stelle kommentierte Beispiel nicht dazu zählt und noch im selben Kommentar zur Ähnlichkeit eines anderen Gleichnisses gesagt wird: „,non è così anacronistica“ (Kenney 2011, ad met. VII, 106-108 (gegen Anderson 1972, ad loc.)). Zu diesem Lemma (anacronismo) s. auch u. S. 162, Anm. 187 (Anachronismen in der Hypodiegese), S. 212, Anm. 205 (Synchronismen) und S. 354, Anm. 633 (Aktualisierungen).

185 Vgl. Barchiesi ${ }^{2} 2013$, CXXVI-CXXIX. Was aber dann Romanisierung und Hellenisierung, außerdem mehrfach simultan genannt, in ihrer konkreten Manifestation im Text genau bedeuten, lassen diese generellen Anmerkungen offen. Wichtig ist jedoch der Hinweis auf den Dualismus aus Rekonstruktion des myth-historischen Kontextes und aktiven funktionalen Aspekten im Verlauf des Gedichts, die der so verstandenen Romanisierung (Hellenisierung wohl weniger) zukommt. Vgl. ebd., CXXVI und CXXIX. 186 Analog zu Landwehrs Aphorismus in puncto Anachronismus. S. o. S. 9, Anm. 1.

187 Vgl. ebd., 179-182. Die Stelle wird so auch bei Barchiesi 2008 besprochen.

188 Vgl. Barchiesi ${ }^{4} 2013$, ad met. I, 168-176. Nicht plausibel ist die Feststellung, dass schon nach dieser Stelle - also met. I, 168-176 - die Anspielungen (!) auf Rom „selten und implizit“ wären, selbst wenn hiermit expressis verbis nur die Stadt als urbaner Siedlungsraum gemeint wäre (zur Erinnerung: bei Wheeler 1999a, 198, ist immerhin met. II, 538f. (kapitolinische Gänse) die in ihrer Behauptung ebenso problematische „last explicitly Roman allusion“). Auch der Aussage, dass Rom in den Büchern XIV und XV das „teatro dell'azione“ (Barchiesi ${ }^{4} 2013$, ad met. I, 168-176) darstellt, ist trotz der Hervorhebung des „finale cesariano“ entschieden zu widersprechen - allein quantitativ mit der KirkeEpisode in met. XIV und der Pythagoras-Episode in met. XV, die in Süditalien oder sicher nicht in Rom spielen. Zur Kritik an der intratextuellen Deutung bei dieser Antizipation in Bezug auf die letzten Bücher s. o. S. 109, Anm. 124.

189 Ebd., ad met. I, 172. 
stichhaltig zu begründen, worin genau die Besonderheit im Gebrauch just des Wortes atria besteht, oder zu erwähnen, dass allein schon Ovid dies in derselben Form - und auch dort, wo der hier zweifellos sehr starke politische Bezug kaum eine Rolle spielt wiederholt in den „Metamorphosen“ verwendet. Einzig die Funktion als ein weiteres Zeichen der Romanisierung, was um einen Verweis auf eine ähnliche, aber nicht das atrium beinhaltende Ennius-Stelle ergänzt ist, scheint bei diesem Wort nennenswert. ${ }^{190}$ Wie oben bei Buchheits Aufsatz schon angemerkt, rückt durch die Priorisierung der politischen Deutung das Ergründen der poetischen Technik eher in den Hintergrund. Folglich fallen die nicht unerheblichen Unterschiede gegenüber ähnlichen, ebenso im Kontext dieser sehr weit gefassten Romanisierung auftretenden Erscheinungen wie Intertextualität, Allusivität oder epischen Gleichnissen definitorisch weniger ins Gewicht. ${ }^{191}$

Im Allgemeinen ist daher das Bild, das sich aus diesem Kommentar zu den zeitlichen Inkonsistenzen der „Metamorphosen“ gewinnen lässt, weder vollständig noch in sich stimmig, weil die einzelnen Textstellen nicht einheitlich und nicht immer mit der nötigen Tiefe besprochen werden. Die anregenden Deutungsansätze und die Hinzuziehung weiterer, vorher unbekannter Beispiele sind aber eine wichtige Ergänzung zum Bömer-Kommentar.

\subsection{Methodische Positionierung}

Die Frage, was bei einem seit Jahrhunderten so intensiv interpretierten und noch stärker rezipierten Text wie den „Metamorphosen“ an Neuem zu Tage gefördert werden kann, ist - so berechtigt sie vordergründig sein mag - letztlich unzulänglich, weil sie impliziert, dass das Alte, bisher dazu Vorgebrachte das Gedicht schon zufriedenstellend erschließen und dessen Charakteristika überzeugend hat erklären können. Direkt auf das hier beachtete Problem der zeitlichen Repräsentation fiktiver Realität bezogen, scheint der Stand der wissenschaftlichen Auseinandersetzung, wie er sich in der nun zusammengetragenen Skizze darbietet, die Problematik dieser Implikation validieren zu können: Weder lässt sich dieser im Jahr 2020 als ein Resultat einer kontinuierlichen Weiterentwicklung und eines fundierten argumentativen Dialogs mit früheren Deutungsansätzen bezeichnen noch stammt ein wesentlicher Teil der hierzu

190 Gerade das macht es umso verwunderlicher, dass in diesem Kontext neben einer kurzen Erläuterung bei valvis apertis im gleichen Vers die anderen sehr markanten Beispiele für Aktualisierungen, die man vor dem Hintergrund der beabsichtigten Rom-Analogie als Romanisierungen par excellence bezeichnen könnte, gänzlich unerwähnt bleiben: bei celebrantur (172), nobilium-plebs (172f.), potentes caelicolae clarique (173f.) und posuere Penates (174). S.u. Kap. 7.1.3.1.

191 Dass dann die anschließende Besprechung von Ovids gleichnishaft-metaphorischer Bezeichnung des Göttersitzes als Palatia caeli (met. I, 176) wiederum sehr umfangreich ist, bestätigt diesen Eindruck sehr gut, da an dieser Stelle die zeitgeschichtlichen Aspekte von großer Bedeutung sind. 
gewonnenen Erkenntnisse aus dezidiert auf diese Fragestellung fokussierten Arbeiten.

Trotz des unübersichtlich großen Umfangs an Altem ist ein Bedarf für Neues deshalb durchaus gegeben, jedenfalls nicht im Vornherein schon zu verneinen. Unter Aufgreifen der vielversprechenden Ansätze, insbesondere des Korpus bei Ebert und Bömer und der Argumentationsweise bei Kraus, von Albrecht, Solodow, Schmidt, Tronchet, Feeney, Wheeler (die letzten drei besonders in Bezug auf den Anachronismus), kann so eine prinzipielle Erfassung erfolgen, deren interpretatorischer Schwerpunkt auf der textimmanenten Beschreibung der Aktualisierungen und Anachronismen als ästhetische Phänomene liegt. Der strukturalistische Zugriff soll die Untersuchung auch der Notwendigkeit entheben, einer im Vorfeld formulierten, im Text zu beweisenden oder zu widerlegenden Hypothese nachgehen zu müssen.

Anstelle der begrifflichen Beliebigkeit und Ungenauigkeit, die sich als vielfältiges Panorama beginnend beim inflationär gebrauchten und wenig spezifischen „Anspielen“192 bis hin zum „chronologischen Widerspruch“ darbieten, und statt einer Deutung, die der Erfassung und Beschreibung des zu Deutenden vorauseilt, soll so eine Einordnung des Textbefundes in die Ästhetik des jeweils vorliegenden Textabschnittes wie auch in die des Gesamtgedichts angestellt werden. ${ }^{193}$

192 Nebenbei bemerkt ist schon bei Servius die Verwendung von alludere oder vergleichbaren Ausdrücken wenig präzise. Vgl. Stok 2016, 423-426.

193 Eine solche Vorgehensweise hat folglich nichts mit einem aus historistischer Sicht missverstandenen „anachronism hunting“ (Cole 2004, 414 (Anm. 142)) gemein, weil es ihr Ziel ja ist, lediglich das zu beschreiben, was da ist; und je grundlegender dies erfolgt, auf desto validere Weise lässt sich ein Urteil darüber bilden - und nicht andersherum. $\mathrm{Zu}$ in dieser Hinsicht „absurden Annahmen als Konsequenz der vertretenen Prämissen“ s. Schmidt 1991, 21. 